\title{
Profesionales y profesores del Urbanismo en el Siglo XX en la F.A.U. de la Universidad de Chile
}

M. Isabel Pavez Reyes

\section{Filiación}

Académica del Departamento de urbanismo de la Facultad de Arquitectura y Urbanismo de la Universidad de Chile.

\section{Resumen}

En el marco de las celebraciones de los 150 años de la Enseñanza de la Arquitectura en la Universidad de Chile y, cuando el Departamento de Urbanismo de la Facultad de Arquitectura y Urbanismo se encuentra celebrando los 50 años desde su creación (experimental 1949 / oficial 1952 - 1999/2002), hacemos un recuerdo de los profesionales y profesores del Urbanismo en nuestra casa de estudios en siglo XX.

\section{Palabras clave}

Profesionales y profesores de Urbanismo en la F.A.U. de la Universidad de Chile, siglo $\mathrm{XX}$.

\begin{abstract}
As part of the celebrations of 150 years of the Teaching of Architecture at the University of Chile and, when the Department of Urban Planning School of Architecture and Urban Design is celebrating 50 years since its creation (experimental 1949 / 1952 official - 1999/2002), we recall the urban planning professionals and professors in our university in the twentieth century.
\end{abstract}

\section{Key words}

Urban professionals and teachers in the FAU University of Chile, the twentieth century.

\section{Sumario}

Introducción

1.-Arquitectos ex alumnos - ex profesores: Galería - Antología.

2.-Profesionales y Profesores del Urbanismo U. de Chile siglo XX

3.-profesionales del Urbanismo, visitantes en F.A.U.

\section{Introducción}

En el marco de las celebraciones de los 150 años de la Enseñanza de la Arquitectura en la Universidad de Chile $y$, cuando al finalizar el siglo XX, el Departamento de Urbanismo de la Facultad de Arquitectura y Urbanismo se encuentra celebrando los 50 años desde su creación (experimental 1949 / oficial 1952 - 1999/2002), hacemos un recuerdo de los profesionales y profesores del Urbanismo en nuestra casa de estudios en siglo $\mathrm{XX}$. 
Cuando observamos el siglo que está concluyendo en lo referido a la relación de los arquitectos de la Universidad Chile con la disciplina del Urbanismo, es claro, en primer lugar, que en todos estos cien años, al menos un grupo de profesionales estuvo especialmente interesado y comprometido en la difusión, cultivo y aplicación de los principios que esta especialidad ha ido sosteniendo a través de las décadas.

Esto ocurrió, estrechamente ligado $-\mathrm{y}$, en ocasiones, a pesar de- un contexto nacional que se ha presentado -tanto desde los aconteceres naturales como institucionalesmás favorable, o menos favorable, según sea el caso y el momento histórico, a la problemática y desarrollo de los asentamientos humanos; y de las transformaciones de las estructuras institucionales universitarias y de sus planes de estudios ${ }^{1}$.

Podríamos caracterizar el siglo, a partir de cuatro períodos, a modo de breve introducción al presente homenaje, a saber: 1900-1928; 1929-1957; 1958-1979; y 1980-2000.

1900, marca la incorporación de la Escuela de Arquitectura a la Facultad de Ciencias Físicas y Matemáticas de nuestra Universidad.

En todo el primer período se abogó tanto en el ámbito nacional como en el latinoamericano, por acciones de previsión del desarrollo de las ciudades, por la realización de planos reguladores en todo centro urbano, por la creación de cursos de Urbanismo en Escuelas de Arquitectura, por la creación de Facultades de Arquitectura y Urbanismo en las universidades, y por la difusión de esta disciplina desde las sociedades de arquitectos.

Diversos arquitectos que viajaron a realizar estudios de arquitectura, y más excepcionalmente de Urbanismo, a Europa y Estados Unidos, aportaron libros, experiencias y también diversas proposiciones, que permitieron animar el debate en arquitectura y urbanismo que se llevaba en el país.

1928 vio la creación del curso de Urbanismo por el Prof. Arqto. Alberto Schade P., en el nivel de pre-grado, y, como consecuencia del terremoto de Talca, Constitución y

\footnotetext{
${ }^{1}$ Puede verse al respecto:

GUROVICH W., Alberto, "Conflictos y negociaciones: la Planificación Urbana en el desarrollo del Gran Santiago", en PAVEZ R., María Isabel, Juan Partrochia Beguin. Premio Nacional de Urbanismo 1996, Departamento de Urbanismo, F.A.U. de la Universidad de Chile, 1998, Sesquicentenario de la Enseñanza de la Arquitectura en la Universidad de Chile, 308 págs. ilustradas, inédito.

GUROVICH W., Alberto, "La venida de Karl Brunner en Gloria y Majestad. La influencia de sus lecciones en la profesionalización del Urbanismo en Chile", en revista DE ARQUITECTURA No8, Especial Karl Brunner, ISSN 0716-8772, Santiago 1996, pp.8-13.

PAVEZ R., María Isabel, La Institución del Urbanismo en la Facultad de Arquitectura y Urbanismo de la Universidad de Chile 1928-1988, Departamento de Urbanismo, F.A.U. de la Universidad de Chile, Sesquicentenario de la Universidad de Chile, 1992, 151 págs.
} 
Curicó, en ese mismo año, también se dictó la primera Ley General de Construcciones y Urbanización.

El inicio del segundo período, en 1929, marca el comienzo de la profesionalización del Urbanismo con la instauración de un Seminario de Urbanismo para post-titulados, por el arquitecto urbanista austríaco Dr. Karl Brunner; un curso centrado en el ámbito del Diseño Urbano.

A la partida de K. Brunner, quien había sido su alumno en Viena, el Prof. Arqto. Rodulfo Oyarzún P. continuó realizando este seminario por algunos años.

La mayoría de los arquitectos ex alumnos de Brunner en Chile realizaron más tarde, aportes docentes en el nivel de pre-grado en nuestra Universidad, entre ellos los Arqtos. Enrique Gebhard P., Guillermo Ulriksen B.; Federico Oehrens D., y su contraparte y colaborador en Chile, Arqto. Luis Muñoz Maluschka.

Cuando la gran crisis económica mundial estaba llegando a Chile en medio de graves convulsiones sociales y políticas, el Arqto. Muñoz, antes mencionado, comenzó sus viajes a Alemania desde 1931, para realizar estudios, prácticas e intercambios que se sostuvieron por más de veinte años con las instituciones y planificadores urbanos y regionales del Estado de Hannover, Baja Sajonia.

La difusión en Chile de los conceptos de ordenamiento territorial adquiridos por Luis Muñoz durante esos años, especialmente a partir de los primeros Congresos Nacionales de Arquitectura, y de Urbanismo, sumada a la efervescencia del debate y exigencia práctica derivada del gran terremoto de 1939 donde tuvo decisiva participación, y la acción del mismo en los siguientes años para efectos de una aplicación más generalizada de esos conceptos en el país, le permitieron aportar, al pasar a la segunda mitad del siglo, la legislación (1953) que reconoció los escalones territoriales de la planificación con sus correspondientes instrumentos e institucionalidad en el Estado, abriendo amplios espacios de participación, tanto para el ejercicio profesional como para la docencia y creación de conocimientos en el nivel superior, durante los siguientes veinte años.

En la segunda mitad de la década de los años 1940s, la enseñanza de la arquitectura se reestructura en función de la Bauhaus, adoptando la carrera de arquitecto el perfil del "arquitecto integral".

La primera mitad de los años 1950s, vio también la titulación en nuestra Facultad, de un grupo de destacados alumnos, entre ellos, Jorge Poblete G., Juan Honold D., Jorge Martínez C., Pastor Correa P., Juan Parrochia B., que pronto comenzaron a tener una labor señera en materia de Urbanismo y Planificación Urbana y Regional en Chile. Simultáneamente se creaba el Instituto de Vivienda Urbanismo y Planeación -hoy Departamento de Urbanismo, e Instituto de la Vivienda-, dando comienzo a una labor de investigación esencial.

El año término del segundo período, 1957, indica la realización del "Seminario del Gran Santiago", evento de carácter interdisciplinario, que dio cuenta de una madurez en la 
conciencia de los problemas urbanos y la voluntad de emprender la pronta solución de ellos en la escala metropolitana y regional emergente, reconocida oficialmente en las importantes leyes establecidas desde 1953. Nuestra institución y sus ex alumnos y profesores tuvieron activa participación en dicho evento.

En la apertura del segundo período, el año 1958 señala la aprobación técnica del Plan Regulador Intercomunal de Santiago, con estudios y proyectos, donde han tenido participación protagónica los Arqtos. Juan Honold D. y Juan Parrochia B., quienes dedicaron también parte de su tiempo a la enseñanza del Urbanismo y la Planificación Urbana y Regional en nuestra casa de estudios, junto con su intensa actividad profesional en las instituciones del Estado.

En 1960, el Prof. Francis Violich, de la Universidad de California, Berkeley, U.S.A., realiza una misión en Chile, patrocinada por el Ministerio de Obras Públicas, presentando un informe donde sostiene la necesidad de una enseñanza interdisciplinaria de posgrado en planeamiento regional y nacional. Esta misión fortalecerá los programas existentes a la fecha en la Universidad de Chile, Universidad Católica de Santiago y Universidad Católica de Valparaíso.

En 1961 se decretó que toda ciudad sobre los 50.000 habitantes debería contar con el cargo de Asesor Urbanista desempeñado por un arquitecto. Simultáneamente, se realizaba en nuestra casa de estudios un importante seminario sobre la enseñanza de la Planificación con la participación de varias Facultades. Una nueva misión, ahora del Prof. John Parker, de la Universidad de North Carolina, U.S.A., incrementó las acciones para reinstaurar un curso de postítulo, dando lugar en 1962 a la proposición de un "Curso Superior de Planificación Urbano-Regional", el que fue impartido desde 1964 y hasta 1979.

La creación en 1965 del Ministerio de Vivienda y Urbanismo, dio una nueva dimensión a la participación del arquitecto en las materias de desarrollo urbano, que desde hacía ya muchos años venían realizándose desde el Ministerio de Obras Públicas. Como repercusión de la creación de dicho Ministerio, nuestra Facultad cambió su nombre por Facultad de Arquitectura y Urbanismo. Casi treinta años habían transcurrido desde que nuestros profesores habían propuesto este nombre y objetivos por primera vez.

La oficialización en 1967 de la Oficina de Planificación Nacional, vino a incrementar la cultura de la planificación en términos de incorporar enfoques integradores de las variables económicas, sociales y físicas. En nuestra unidad académica asumía en este mismo año la dirección del IVUPLAN, el Prof. Arqto. René Urbina V., en momentos en que la F.A.U. se centraba en revisar sus objetivos dentro de la Universidad.

Durante la década de los años 1960s, en medio de reformas a todo nivel en el país, se había cuestionado la formación del "arquitecto integral", desplazándose el interés hacia la instauración de las especialidades en la enseñanza. Los profesores de Urbanismo, inspirados también por la Declaración de Delos (1963), llegaron a postular hacia el final de la década, un Departamento de Organización Ambiental, e incluso, una "Facultad de Organización Ambiental", según observamos en una ponencia del Prof. Arqto. Ronaldo Ramírez R. 
De igual forma, hacia fines de la década IVUPLAN presentó un "Programa de Desarrollo de Ciencias Urbano-Regionales y Planificación", en el marco de un convenio entre la Universidad de Chile y la Universidad de California, que en el caso de la Universidad de Chile comprometió a varias Facultades; Arquitectura y Urbanismo, Ciencias Económicas, Ciencias Jurídicas y Sociales, y Filosofía y Educación.

Entre 1968 y 1973, el Prof. Arqto. Fernando Kusnetzoff K., se desempeñó como Decano de nuestra Facultad.

En el medio externo, en la misma década, y hasta comienzos de los 70s, el Prof. Arqto. Juan Parrochia siguió el camino de la implementación del Plan Intercomunal de Santiago, con el despliegue de los sistemas estructurantes de equipamientos y de circulación metropolitanos, realizando la vialidad estructurante de la intercomuna y el Metro de Santiago, entre otros llevados a cabo desde el Ministerio de Obras Públicas. Ello significó la apertura de una nueva subespecialización en materia de Urbanismo en Chile, la del Transporte Urbano, colaborando indirectamente a crear la institucionalidad para su enseñanza en la Universidad de Chile y posteriormente en la P. Universidad Católica de Chile.

En 1970, el IVUPLAN se convirtió en Departamento de Planificación Urbano-Regional, elaborando un Plan Departamental de Diversificación Profesional (1973-77), que incluía bachilleratos, grados técnicos, perfeccionamientos y especializaciones y Maestría y Doctorado en planificación del desarrollo urbano. El programa, aprobado, no fructificó en la práctica, y se detuvo definitivamente a raíz de los sucesos nacionales de 1973.

A partir de este año y hasta 1975, cumplió funciones de Decano el Prof. Arqto. René Martínez L., realizando una misión moderadora relevante en los primeros dos años, especialmente difíciles, de la intervención del gobierno militar en la Universidad de Chile.

El año 1979, señaló el término de la participación del Estado en gran escala en materia de desarrollo urbano en Chile, el comienzo de la desestructuración del modelo económico precedente, y el fin de la etapa de industrialización por sustitución de importaciones.

La política Nacional de Desarrollo Urbano de 1979, trajo consigo la cancelación de las normas que modelaron por mucho tiempo el espacio habitable, para desencadenar la regulación por la competencia mercantil, lo que marca todo el último cuarto del siglo $X X$.

En 1981, el Departamento de Planificación Urbano-Regional, pasó a denominarse Departamento de Urbanismo, creándose en 1985, el Magister en Urbanismo en nuestra Facultad, donde participaron varios de los ex alumnos ex profesores citados en este homenaje.

Finalmente, podemos señalar que, especialmente en los 50 años transcurridos entre el inicio del segundo período señalado, 1929, y el término del tercer período, 1979, los ex 
alumnos y ex profesores a quienes rendimos homenaje en este escrito tuvieron, por una parte, una participación relevante en materia de desarrollo urbano en el país, y, por otra, entregaron su experiencia y conocimientos con gran generosidad en la docencia superior en nuestra casa de estudios.

\section{1.- Arquitectos ex alumnos - ex profesores de Urbanismo}

Rendimos un sencillo homenaje a los arquitectos ex alumnos - ex profesores, responsables de asignaturas de Urbanismo del siglo $\mathrm{XX}$, mediante una selección de breves extractos de su obra escrita.

Las fotografías que acompañan esta antología, se han encontrado en los archivos de la Secretaría de Estudios, del Departamento de Urbanismo y del Departamento de Historia y Teoría de la Arquitectura de la F.A.U. Universidad de Chile; en revistas nacionales, en especial, ZIG-ZAG, ARQUITECTURA, URBANISMO Y ARQUITECTURA, AUCA, CA, DE ARQUITECTURA; en publicación de la UNIVERSIDAD DEL BIO-BIO, y en archivos privados.

\section{Galería - Selección antológica}

\section{Entre otros:}

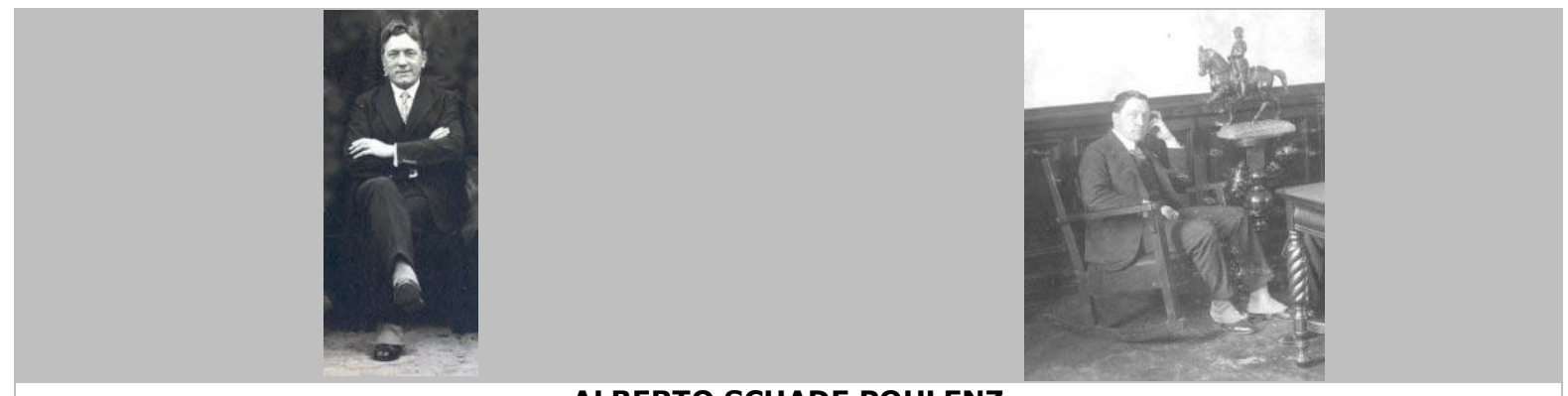

\section{ALBERTO SCHADE POHLENZ}

[1922] "Hace votos [el suscrito, A. Schade P.]: $1^{\circ}$.Porque las autoridades nacionales y locales de todos los países de América legislen en forma práctica y de perentoria obligación inicial, el estudio y la adopción de planos reguladores de todo centro urbano, recomendándose que sólo por excepción y en zonas relativamente pequeñas se siga el sistema de cuadrículas uniformes que ha predominado hasta ahora; se determine el emplazamiento, disposición y extensión de los parques, jardines plazas y carácter de sus plantaciones $y$ otros espacios libres que tengan por objeto la higienización interior de las manzanas; se prevea la ubicación conveniente de los edificios públicos y de los monumentos, y como complemento necesario se formule un cuerpo de disposiciones que reglamenten la aplicación de los planos en todos sus aspectos; $2^{\circ}$ Porque las Facultades y Escuelas de Arquitectura, incluyan en sus programas un curso especial de urbanización y las sociedades de arquitectos creen clases libres y gratuitas para la divulgación de sus principios. $3^{\circ}$. Porque se constituyan, por iniciativas de las sociedades de arquitectos en cada ciudad de América, 'Ligas', con el fin de despertar, dirigir y estimular la iniciativa oficial en los problemas importantes del plan orgánico de los centros urbanos; $4^{\circ}$. Porque se funde una 'Liga Panamericana de Ciudades' ".

Fte.: Extracto de "Conferencia del Señor Alberto Schade P., Presidente de la Sociedad Central de Arquitectos, en Revista de Arquitectura, Sociedad Central de Arquitectos de Chile, $\mathrm{N}^{\circ} 1$, mayo de 1922, Editorial F. Trouve, pp.5-7 y continúa en pp.17-20. 


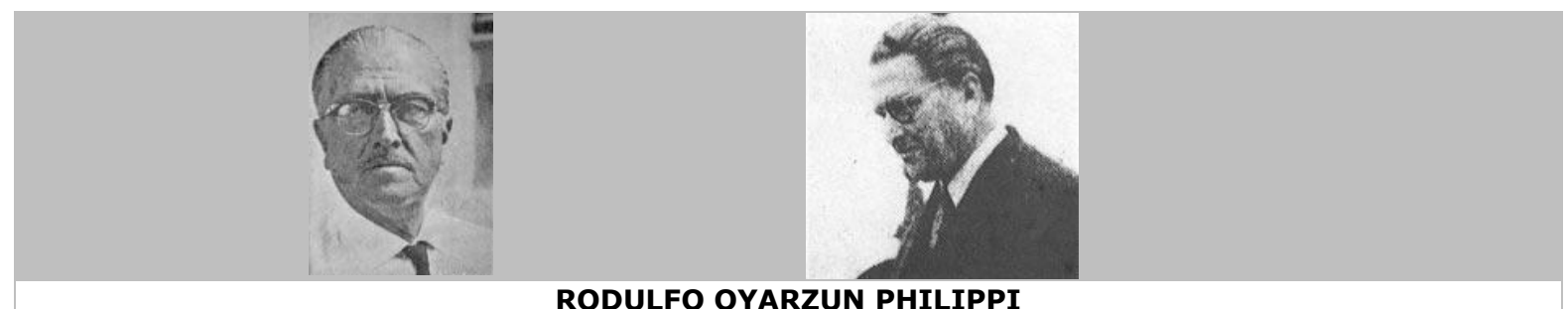

[1970, refiriendo sobre años 1920s] "A pesar de que nuestro ambiente latino-americano no era propicio al urbanismo, de que nuestras ciudades vivían su etapa post-colonial, pre-maquinista, de que la arquitectura se debatía en plena decadencia, y de que, en materia de viviendas, se comenzaba recién a vislumbrar el valor básico de su función celular, nacía el deseo fervoroso de innovación, especialmente entre los arquitectos. No fue para Chile una mera coincidencia que estas aspiraciones se acrecentaran, ya que el año 1920 dejábamos de ser el último rincón del mundo, a raíz de la apertura del canal de Panamá. Y cupo a Chile destacada actuación en la organización de los Congresos Panamericanos de Arquitectos, en que Ricardo González Cortés iniciara su carrera brillante en defensa de la arquitectura y el urbanismo. En estas reuniones predominó desde la partida el interés unánime para resolver los problemas relacionados con el desarrollo de la ciudad y su crecimiento, el estudio racional sobre el futuro de la vivienda americana, con la creación de las Facultades de Arquitectura, y la importancia de la profesión de arquitecto, y de la enseñanza del urbanismo. Es admirable constatar que, dentro de un formalismo romántico de banquetes, paseos oficiales, discursos ampulosos, de acuerdo con las costumbres de la época, se impusiera un criterio que permitiera sembrar ideas básicas que, dentro de pocos años, fructificarían en valiosas realidades".

Fte.: OYARZUN PH., Rodulfo, "El Prof.Dr. Karl H. Brunner. Su estada en Chile entre los años 1929-1934", en: REVISTA DE PLANIFICACION, Vivienda Ciudad Región, N7, 1970, IVUPLAN, F.A.U. Universidad de Chile, pp. 139-153.

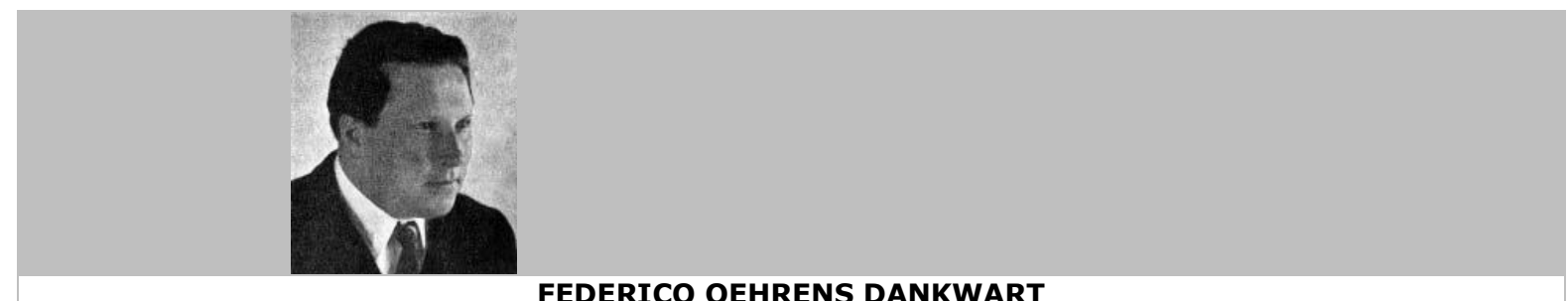

[1939] "Ha llegado el momento en que es necesario crear ciudades próximas a las faenas agrícolas, ciudades que puedan servir de elementos de unión entre obreros y campesinos que en ellas vivan o trabajen, y habilitar también, simultáneamente, ciudades ya existentes, tendiendo buenas vías de comunicación a los campos para que los campesinos puedan vivir en contacto más inmediato con el progreso material y cultural. Estas ciudades se dotarían de servicios de urbanización de primer orden. Es indudable que no hay otra manera de sacar de su estado rudimentario al campesinado".

Fte.: Extracto de: OEHRENS D., Federico, "Sobre el problema de antagonismo entre la ciudad y el campo", en revista URBANISMO $Y$ ARQUITECTURA, N04, $2^{\circ}$ sem. 1939, pp. 29-34. 


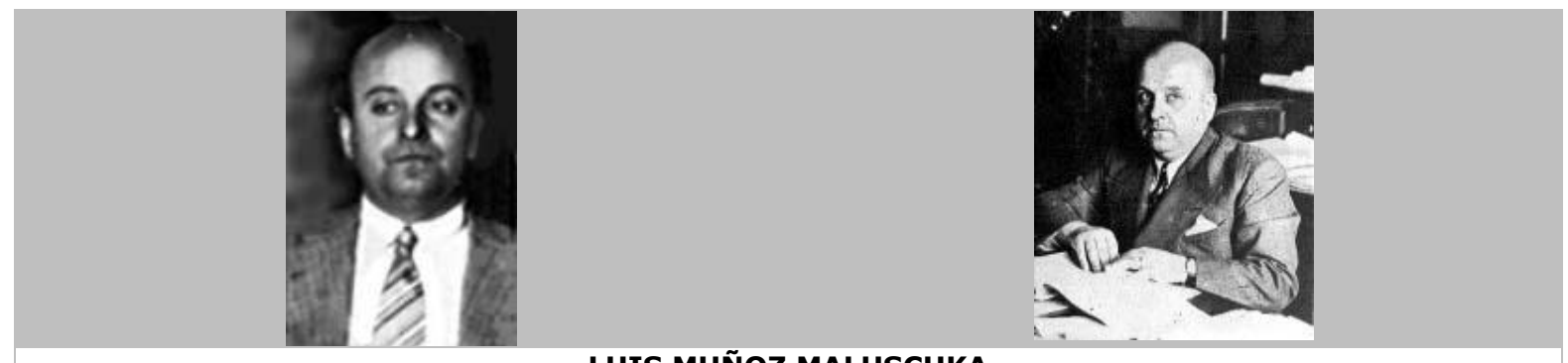

\section{LUIS MUÑOZ MALUSCHKA}

[1936] "La administración comunal no tiene influencia ni medios para intervenir en forma efectiva en la determinación del desarrollo o incremento de la vialidad territorial, a pesar que, para ella, se trata de un problema de vida o muerte. Sin embargo, consideramos indispensable que el interés comunal se haga presente en el momento que se establezcan los planes de incremento vial, que, como hemos dicho, determinan el desarrollo futuro de los núcleos poblados. Esta influencia sólo puede lograrse si se llegara a establecer verdaderas FEDERACIONES INTERCOMUNALES DE PLANEAMIENTO ECONOMICO TERRITORIAL, que le darían a los diversos núcleos poblados, cierta estabilidad en su función económica y, por lo tanto, una previsión para su desarrollo futuro. Consideramos indispensable que las diversas comunas que pertenecen a una región de características económicas definidas, se federen y establezcan verdaderas "Asociaciones de Planeamiento Territorial", buscando un entendimiento para resolver los problemas económicos de interés común. (...) Los problemas de carácter intercomunal que le correspondería resolver a cada central de planeamiento, son organizar las actividades económicas del territorio, mejorando la vialidad entre los centros de trabajo y los de residencia en general, entre centros de extracción y los de elaboración, como asimismo entre los de elaboración y los de consumo. Les correspondería además, establecer una política intercomunal definida, referente al incremento de los centros de elaboración, los de recreación y turismo, los administrativos, culturales, financieros y comerciales, y la distribución de áreas de abastecimiento, etc. En esta forma, los planos reguladores serían la representación gráfica de una política de fomento definido y prolongado a través del tiempo, con la finalidad ulterior del incremento económico integral de una región del país. Mientras los diversos municipios no comprendan y dominen los problemas económicos intercomunales que los ligan, y que, dentro de la incomprensión existente, sigan combatiendo y anulándose recíprocamente, no habrá progreso integral, y sólo prosperará la región o ciudad favorecida temporalmente por una influencia política pasajera".

Fte.: Extracto de: MUÑOZ MALUSCHKA, Luis E., "Planos Reguladores y Zonificación Territorial", en: revista Arquitectura, editorial Antares, enero de 1936, pp.20-21.

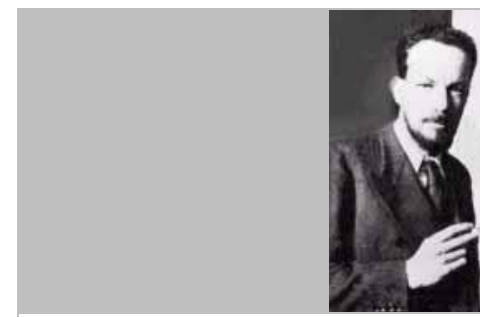

ENRIQUE GEBHARD PAULIS

[1937] "Es evidente que la necesidad de planificar, de organizar, es una consecuencia ineluctable de los tiempos modernos. Es la compensación de la vida actual, caótica y atormentada, por el maquinismo después del 50. La velocidad de la técnica ha generado problemas nuevos en el espacio urbano limitado. Salvo en las postrimerías de la civilización romana, el hecho de la ciudad con más de un millón de habitantes es un acontecimiento novedoso para la humanidad. Ha nacido un organismo con nuevas necesidades, que revoluciona totalmente las costumbres seculares, con nuevas exigencias en el terreno sociológico y que obliga a una revisión cuidadosa de los conceptos de velocidad, tiempo y espacio. La movilización urbana, las arterias vitales de circulación que permiten una clasificación de la velocidad, constituyen una de las funciones primordiales de la ciudad que nace. La calle permite la simultaneidad en el tiempo y éste es la realización del espacio. La salvación funcional del hombre, su evolución en la jornada cíclica de 24 horas de la jornada solar, está en la justa valoración de la colectividad, es decir en el reemplazo de la estética gremial o del artesano por la dinámica de la máquina, concebida como prolongación del hombre y de su tiempo".

Fte.: Extracto de: GEBHARD P., Enrique, "Primer Congreso Chileno de Urbanismo", en: revista Zig-Zag, Edición extraordinaria: Arquitectura - Construcción - Urbanismo, Santiago de Chile, diciembre de 1937. 


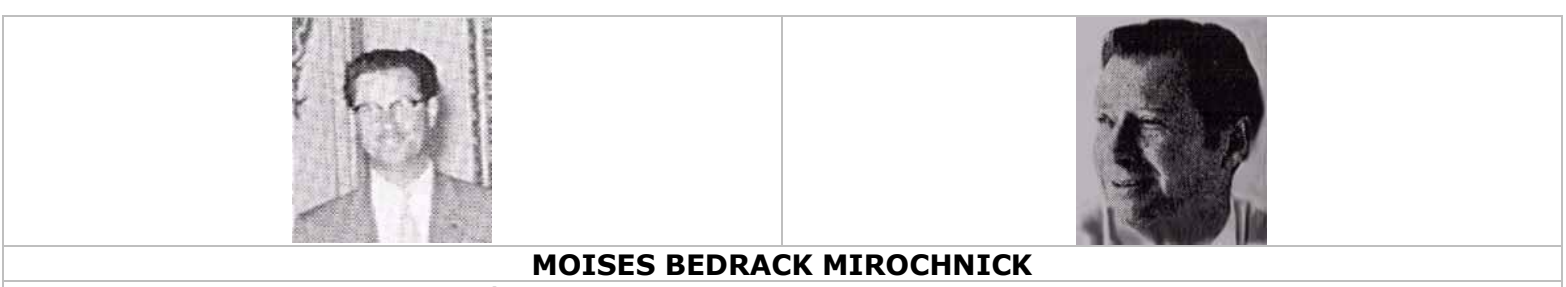

[1972] "En lapso de los dos últimos decenios, en Chile, hemos presenciado auges regionales y su declinación después de 3 ó 4 años, debido a insuficiencia en los estudios de factibilidad, a motivaciones políticas inconducentes y, a la propia inexperiencia con que se aplicaban los sistemas de planificación. (...) La dinámica de cambios ocurrida a través de estas acciones aisladas [Arica 1954-58; Iquique 1960-64; Punta Arenas, 1956] significó fuertes inversiones en nuevas instalaciones y servicios, con la consiguiente subutilización de tales recursos una vez que se empezaron a producir restricciones a los proyectos iniciales, debido a cambios en la orientación política de las administraciones sucesivas. La mano de obra flotante, que se había radicado en estos lugares durante el auge de estas iniciativas, se vio compulsada a buscar nuevas fuentes ocupacionales en otras áreas del territorio.

Estos ejemplos deben conducirnos a dos conclusiones directrices para este Seminario:

i) Estas acciones aisladas y sistemáticas conllevan un costo social que los países en desarrollo deben ponderar si están en condiciones de sostener a mediano y largo plazo, dentro de un balance positivo.

ii) Sin pretender el equilibrio demográfico dentro del territorio como una meta inicial, pensamos que en los modelos de inversión la variable demográfica debe ser manejada en conjunto con la cuantía de los recursos y las metas de crecimiento.

Estas palabras introductoras no pueden concluir sin reseñar las metas y objetivos que el Gobierno Popular chileno, en la vía de construcción del Socialismo, está trazando en relación con la temática de este Seminario. No compartimos la antinomia de vida urbana y vida rural como dos niveles contrapuestos en cuanto al acceso a formas completas y dignificadoras del habitat y modos de producción. Por una parte, la profunda Reforma Agraria que está en marcha, lleva implícita tanto la actividad industrial derivada, como la creación de villorrios agrícolas que permitan concentrar niveles adecuados de servicios básicos. Estos factores pueden considerarse como elementos de arraigo en las zonas agrícolas que deberían desacelerar los flujos migratorios tradicionales. Además, la propiedad social del sector minero representa, en plazos prudentes, el reemplazo de los campamentos por ciudades nuevas o la revitalización de algunas de las existentes, dentro de radios de distancia aceptables, que generarán verdaderas condiciones de vida urbana, aun en las zonas del desierto chileno, creando mayor estabilidad en la población y, en particular en la juventud. Como la agricultura y la minería constituyen parte significativa de la economía nacional, es claro que las medidas de política urbana y habitacional antes indicadas, podemos considerarlas como una respuesta inicial al desafío que, hacia fines del siglo, nos pueden llevar a concentrar al $90 \%$ de la población en ciudades, pueblos, aldeas y villorrios con sus estructuras físicas ya debidamente acondicionadas".

Fte.: BEDRACK, Moisés, "Desarrollo Urbano y Vivienda. Bases políticas para enfrentar el rápido crecimiento demográfico de las ciudades", ponencia al Seminario Técnico OEA-BID Urbanización y crecimiento demográfico en América Latina, Río de Janeiro, abril 3-7 de 1972. Publicada en Chile por la Dirección del Planificación del Desarrollo Urbano, con el título Desarrollo Urbano y Vivienda, 51 págs. 


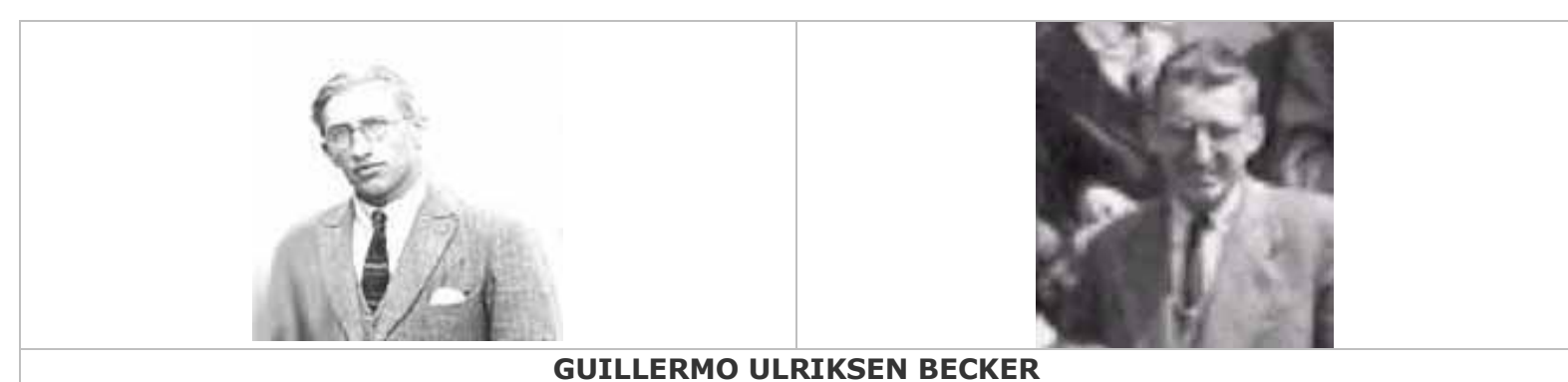

[1953]"Los planificadores deben tener el conocimiento directo de la región (práctica) y deben estudiar las bases científicas (teoría). Sin conocimiento personal de la geografía, población, agricultura, ganadería, minería, comunicaciones y transportes de una región, ningún planificador podrá ser capaz de elaborar un Plano Regulador Urbano. Exclusivamente en base de Geografía Física se han elaborado en nuestro país los planos reguladores urbanos. Sobre aquella base fue elaborado hace veinte años el Plano Regulador de la Comuna de Santiago [Plano Oficial de Urbanización de la Comuna de Santiago, 1934/39, Karl Brunner]. Dieciocho años transcurridos desde su promulgación han evidenciado que las previsiones de este plano eran precarias. La expansión real de la ciudad amenaza de asfixia a la Comuna de Santiago. Es sabido que no se estableció ni siquiera un esquema de plan del futuro desarrollo de la vialidad en la Región de Santiago en aquella oportunidad; de ahí el actual embotellamiento de la Carretera Panamericana en su acceso norte a Santiago. La experiencia de la planificación demuestra que habría sido más fácil establecer un buen plan regional de Santiago, hace veinte años; que [en] un buen plan urbano, el grado de exactitud es más difícil de establecer a medida que se restringe el área de planeamiento. Ello se debe a que los fenómenos económicos se densifican en el mismo grado en que crece la densidad de la población; las mallas del catastro son más estrechas, los precios unitarios del suelo se hacen más elevados, los problemas de planeamiento se hacen más complejos. De ahí la importancia de las tentativas que se hagan para establecer en primer lugar Planes Regionales".

"Esta tentativa [tesis de título origen de este extracto] de establecer bases para el planeamiento del Norte Chico, se plantea como un aporte personal del autor, como fruto de los contactos con los problemas más candentes de la región. Sostengo que no puede elaborarse un planeamiento claro sin una finalidad clara. La finalidad del planeamiento sólo puede emanar de un plan perfectamente claro de desarrollo de la economía que consulte, como una parte de él, la nacionalización de las explotaciones mineras en manos extranjeras $y$, además, una profunda reforma agraria, conjuntamente con medidas de orden financiero, educacional, etc. De ahí que esta tesis se limitará a estudiar las bases para un planeamiento regional. Sostengo, además, que una Junta de Planeamiento no podría formular un plan para el Norte Chico con la perspectiva de algunos años, porque durante la elaboración de ese plan se descubrirán grandes lagunas en el conocimiento científico de esa región lo cual impedirá la formulación de ese plan regional. El autor se propone señalar algunas lagunas en el conocimiento científico de esa región, que pudo constatar en su labor de seis años en el terreno y a través de las discusiones en el seno de pequeñas reuniones de planeamiento. El autor tratará de formular aquellas deducciones de sus observaciones que complementen el conocimiento actual de la geografía, demografía, biología, producción y desarrollo de los recursos naturales de esta región. También ha de repetir nociones elementales sobre dichos temas, pero tratará de ilustrar esas nociones conocidas con ejemplos nuevos. Tiene el propósito de restringir el informe dándole el carácter de una aportación complementaria al conjunto de observaciones y conocimientos que autores tan versados como Fuenzalida, Vila, Matthey, Opazo, Reiche, Brueggen, Fenner, Monsalve, Montalva, Bennett, Harnecker, Ochoa, Zepeda, Frei, Ocampo, Videla Lira, Benítez, Caro y Chouteau, han señalado ya como característico de la geografía económica del Norte Chico. En las conclusiones, el autor espera poder superar esa categoría de aportación complementaria y lograr formular un programa sencillo que sirva de 'aidememoire' de los futuros planificadores del Norte Chico". (...)

"Los verdaderos pioneros de una reforestación inteligente del Norte Chico han sido Sergio Irarrázaval (propietario) y Oscar Prager (paisajista)". (...)

Fte.: Extracto de: ULRIKSEN B., Guillermo, Bases para una Planeación Regional del Norte Chico. Provincias de Atacama y Coquimbo (Región de los Valles transversales), Tesis para optar al Título de Arquitecto, Facultad de Arquitectura y Urbanismo (sic), Universidad de Chile, Profesor Guía: Santiago Aguirre del C., julio de 1952, pp.Programa 1-2; p. Programa 2 ; y p. Flora 25. 


\begin{tabular}{|l|}
\hline VENTURA GONZALEZ MONTECINOS \\
\hline \\
\hline
\end{tabular}

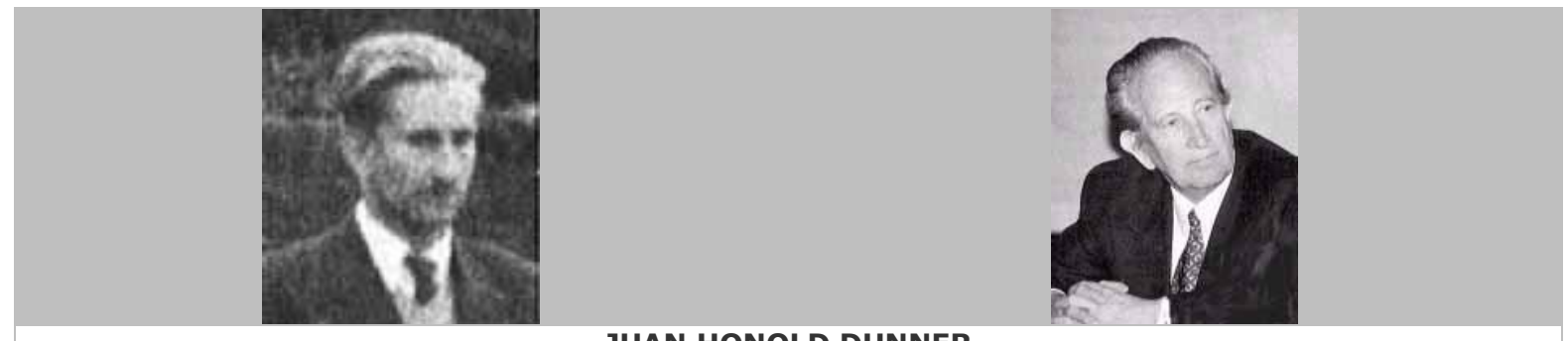

\section{JUAN HONOLD DUNNER}

[1957-58] "El planeamiento de cualquier ciudad, además de los estudios del área propiamente urbana, debe ser necesariamente dirigido a la región en que dicha ciudad se encuentra: de ella provienen los materiales utilizados en su estructura física, de ella provienen los abastecimientos para la vida de sus habitantes y ella le proporciona el marco de su paisaje caracterizador. En el caso de una metrópoli como Santiago, la ciudad ejerce su influencia más allá de la región geográfica y se proyecta en todo el territorio nacional y aun alcanza la escala internacional y continental. Como lógica contrapartida, la metrópoli se relaciona y depende en sus procesos sociales, económicos y culturales, del país entero. Por ello, el programa del Seminario

[Seminario del Gran Santiago, 1957-58] ha recalcado las relaciones e interdependencias del Gran Santiago con los fenómenos territoriales y regionales". (...)

"SÍNTESIS. La complejidad y magnitud de los fenómenos metropolitanos y esta rápida visión de algunos de los problemas más importantes, no deben impedirnos para resumir ciertas ideas centrales, que estamos seguros, serán precisadas y ampliadas durante este Seminario:

1. El proceso metropolitano del Gran Santiago es factor decisivo y principal en el desarrollo social y económico del país. Por lo tanto, la solución de sus problemas debe ser encarada a través de una política derivada del planteamiento territorial y regional de nuestra realidad social, económica, cultural e institucional.

2. La solución del problema urbano no sólo deberá basarse en medidas y planes a realizar en el interior del área urbana, sino que también y fundamentalmente en la organización y planeamiento de la región, única media que hará posible una efectiva descongestión de la ciudad hipertrofiada, mediante el desarrollo de un sistema regional de satélites y áreas de renovación económica.

3. En lo que se refiere a la solución de los problemas urbanísticos, deberá tomarse en cuenta que el área metropolitana es una unidad urbana, y que como tal, posee problemas comunes que deben ser resueltos en conjunto, salvando los límites comunales existentes en la actualidad. Existen diversas fórmulas para encarar el gobierno metropolitano; sin embargo, para el caso de nuestra organización institucional, parece fundamental insistir en la conveniencia de mantener y desarrollar la autonomía municipal establecida en nuestra Carta Fundamental y en la Ley de Municipalidades, y, a la vez, deberá procurarse una fórmula que permita coordinar los complicados procesos metropolitanos en su conjunto.

4. La realización de las obras de urbanismo y servicios públicos que el Gran Santiago necesita requieren la adopción de una serie de medidas específicas para el área metropolitana, de orden financiera, legal y administrativa. Existe urgencia en legislar en eses sentido, por cuanto cualquier nuevo plan técnico urbanístico estará condenado al fracaso si no se proveen las herramientas básicas antes mencionadas".

Fte.: HONOLD D., Juan, Relator, Arquitecto en la Dirección de Planeamiento del MOP. Extracto de "Visión General de los Problemas del Gran Santiago", en: UNIVERSIDAD DE CHILE - BOLETIN INFORMATIVO DEL DEPARTAMENTO DE EXTENSION CULTURAL, N³4, Año III, octubre de 1958. Número especial dedicado a la $2^{a}$ y $3^{a}$ etapa del Seminario del Gran Santiago, 326 págs.; extracto en pp.19-33. 


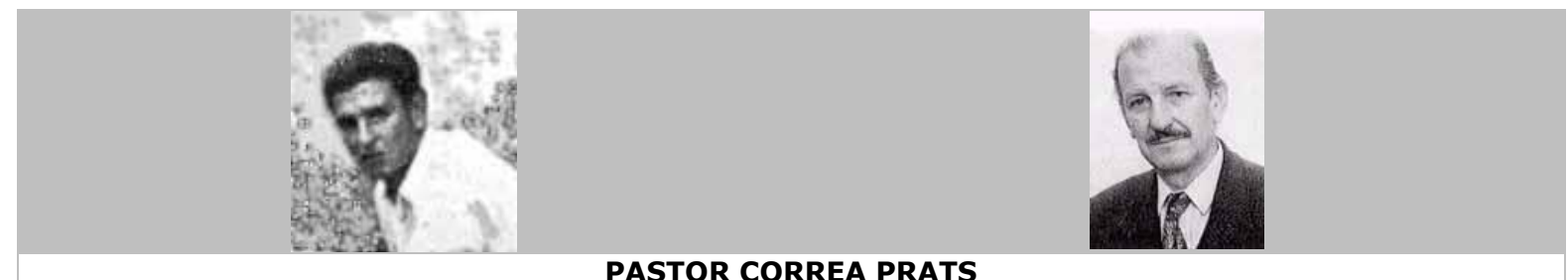

[1957-58] "PROPOSICIONES.

\section{PASTOR CORREA PRATS}

Creación de un Concejo Intercomunal destinado a resolver los problemas comunes a los distintos municipios que forman el Gran Santiago.

Aplicar la clasificación de industrias y de tipos de zonas industriales que se analizan y proponen en el trabajo "Estudio de la Radicación Industrial en la Intercomuna de Santiago", y que aparecen transcritas en el Anexo del presente informe.

Estudiar y aplicar una nueva zonificación de áreas industriales a base de las clasificaciones antes anunciadas y a las consideraciones de orden urbanístico necesarias a saber:

Reglamentar el aislamiento de las zonas industriales exclusivas por medio de franjas de área verde de una profundidad media de 50 mts.

Impedir la interferencia de la vida vecinal y comunal en las zonas mixtas de viviendas con industrias; provocada por el tránsito a que éstas dan origen.

Ordenar y simplificar el sistema vial que relaciona la vivienda obrera con la industria y ésta con la llegada de materia prima y salida de productos elaborados.

(Esta labor deberá seguirla realizando, por mandato del artículo 10, del D.F.L. No 224, la Dirección de Planeamiento del M.O.P., como parte del Plano Regulador Intercomunal, mientras se forme el Concejo Intercomunal propuesto anteriormente).

Comenzar los estudios técnicos sobre contaminación ambiental, tanto del aire como del agua y estudiar la legislación que permita su control. Mientras se forme el Concejo Intercomunal propuesto, esta materia deberá ser abordada por el Departamento de Higiene Industrial del S.N.S.

Estudiar una legislación sobre accidentes del trabajo, enfermedades profesionales y no profesionales que ponga el énfasis en lo preventivo.

Aumentar el actual porcentaje del $5 \%$ de las utilidades industriales, destinadas a construcción de viviendas para obreros, debiendo éstas ubicarse en la misma comuna donde se encuentra la industria para que la relación vivienda-trabajo sea lo más inmediata posible, evitándose desplazamientos innecesarios de la misma masa obrera.

Aplicar una legislación de mayor efectividad que el Decreto No 375, del 27-VII-53, proporcionando a la industria los incentivos necesarios para que se instale en otras zonas del país. (...)

Creación de un Organismo Planificador Estatal, cuya misión sea la de coordinar y orientar el desarrollo integral del país".

Fte.: Extracto de CORREA P., Pastor, Presidente Comisión $N^{\circ} 6$ : Problemas inherentes al trabajo y la producción, "Informe de la subcomisión de industrias sobre radicación industrial en el área intercomunal del Gran Santiago", en: UNIVERSIDAD DE CHILE BOLETIN INFORMATIVO DEL DEPARTAMENTO DE EXTENSION CULTURAL, №34, Año III, octubre de 1958 . Número especial dedicado a la $2^{a}$ y $3^{a}$ etapa del Seminario del Gran Santiago, 326 págs.; extracto en pp.124-132. 


\section{RUVSTADE URBANISMO
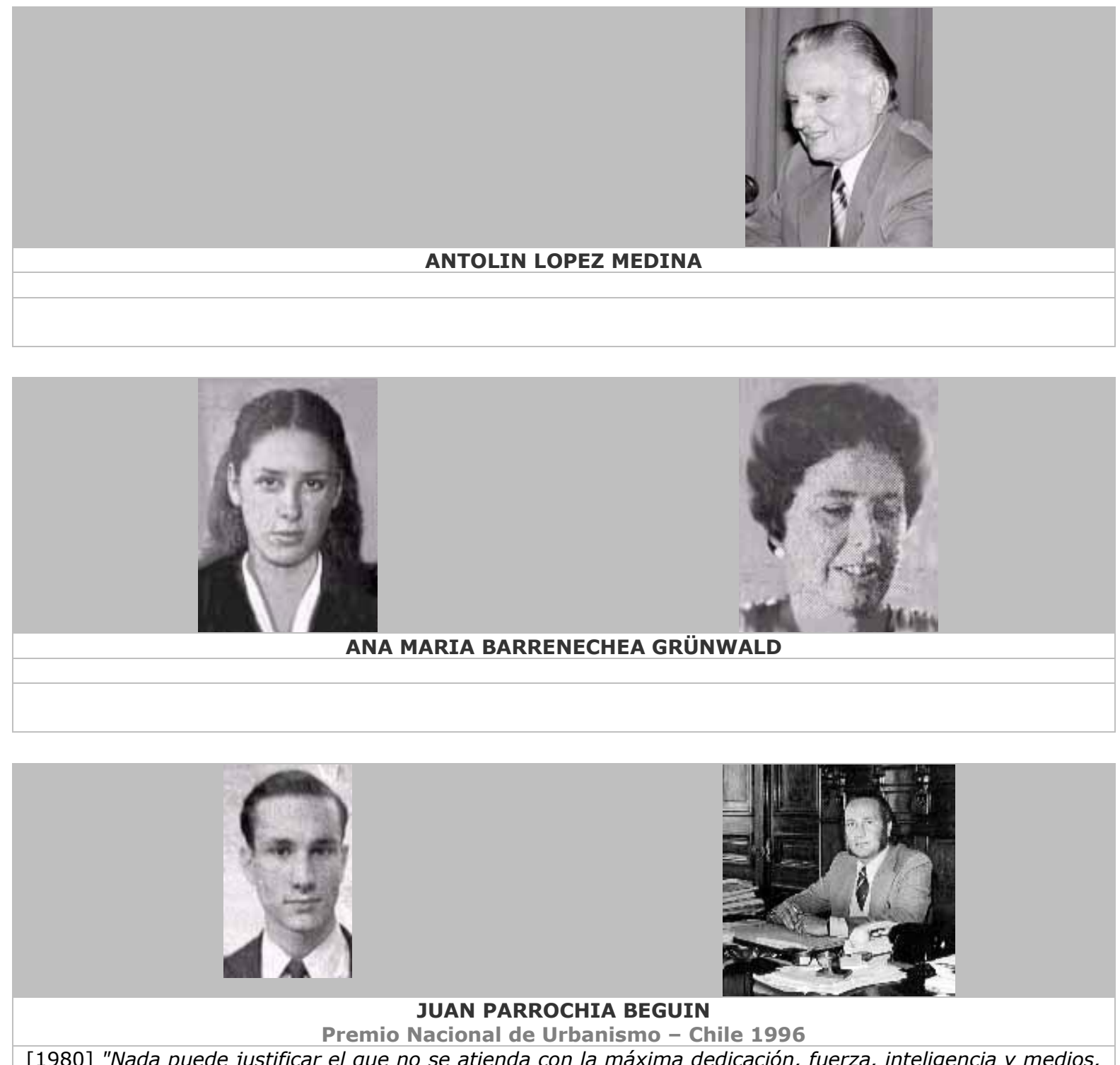

[1980] "Nada puede justificar el que no se atienda con la máxima dedicación, fuerza, inteligencia y medios, los problemas que afectan tan hondamente a todos y cada uno de los habitantes, para darles satisfacción y bienestar, y alentar sus existencias tan plenas de insatisfacciones y dolencias. Esta labor sólo puede trascender al infinito en la medida que nos liguemos en una cadena infinita a través del tiempo con nuestros antepasados y antecesores, y con nuestros descendientes y sucesores, $y$, simultáneamente, en otra cadena también infinita, con nuestros vecinos y compatriotas, y con nuestros coetáneos de todo el mundo. Así se integran formando una cruz, dos grandes equipos de trabajo interdisciplinario, a través del tiempo y a través del espacio. Cada ruptura que se produzca es un paso menos en la obtención de los valores fundamentales y en el desarrollo del género humano".

Fte.: Extracto de: PARROCHIA, Juan, Santiago en el tercer cuarto del siglo XX: El transporte metropolitano en Chile, realizaciones de Metro y Vialidad Urbana, Edición del Departamento de Planificación Urbano - Regional, Facultad de Arquitectura y Urbanismo de la Universidad de Chile, Santiago, 1980, 237 págs. Ilustradas. 

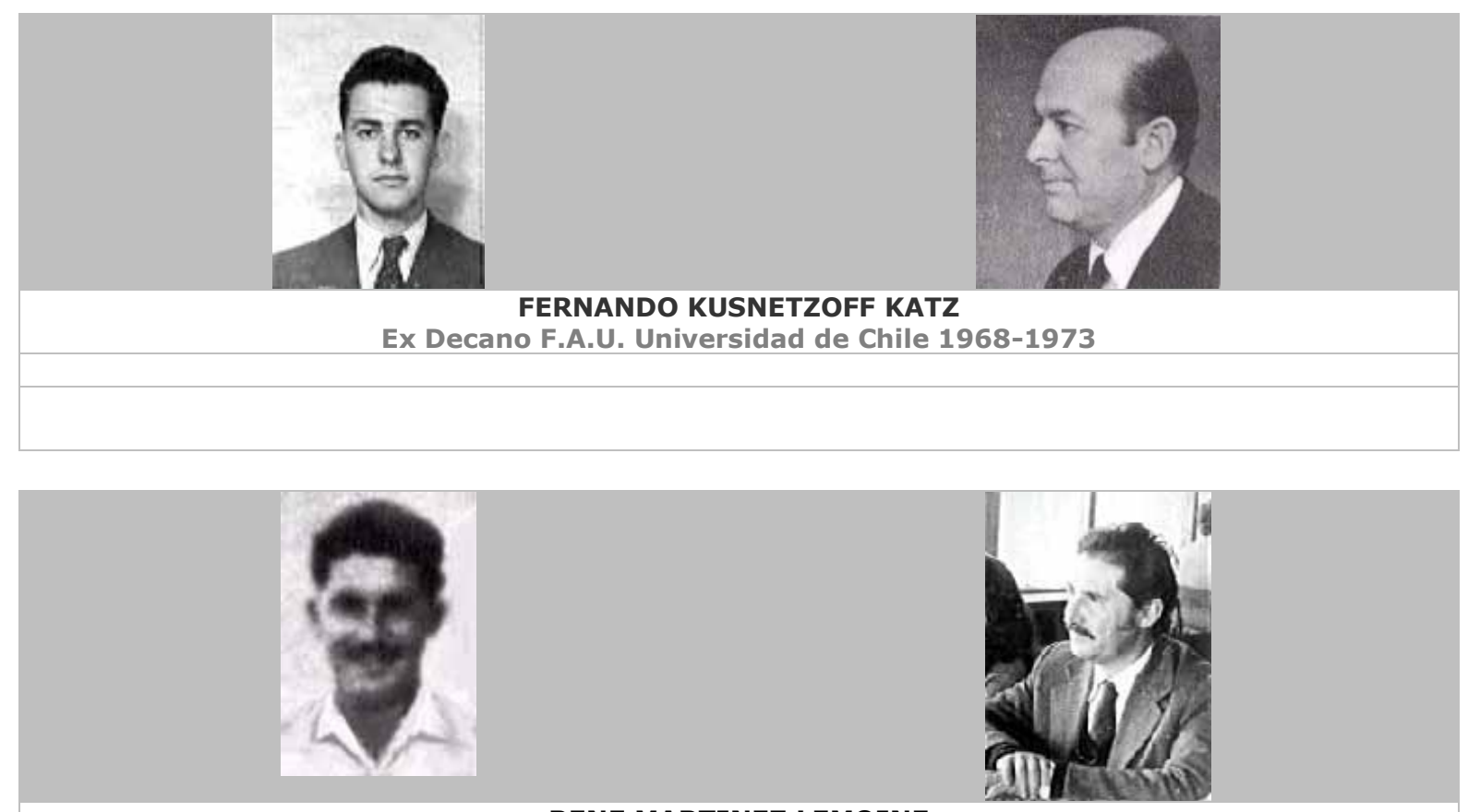

\section{RENE MARTINEZ LEMOINE}

Ex Decano F.A.U. Universidad de Chile 1973-1975

[1977]"Así, sostenemos que el modelo clásico de la ciudad colonial hispanoamericana constituye la culminación de un proceso americano, independiente de disposiciones legales. El modelo así establecido, codificado por la costumbre y convertido en el epítome de la ciudad americana, se proyecta en el tiempo y más allá de la legislación definitiva de 1573, continúa reglando las fundaciones posteriores aún en notable contradicción con disposiciones precisas de la ley. Para fundamentar nuestra tesis, intentaremos un análisis de las disposiciones legales en cuestión relacionándolas con la situación real y cronológica de la fundación de ciudades y con el proceso de dictación, recopilación, ordenación y codificación de las llamadas Leyes de Indias. Nuestro propósito es señalar que los numerosos intentos de recopilación y codificación, la mayor parte de ellos frustrados o no publicados por diversas causas, constituyen al mismo tiempo un esfuerzo por establecer un código orgánico para la vasta empresa civilizadora de España en América y una demostración fehaciente del desconocimiento general de la ley que caracteriza, por razones obvias, a los primeros tiempos de la conquista".

Fte.: Extracto de MARTINEZ L., René, El modelo clásico de ciudad colonial hispanoamericano, edición del Departamento de Planificación Urbano-Regional, Facultad de Arquitectura y Urbanismo de la Universidad de Chile, 1977, 105 págs., extracto en p. 10. 
-

\begin{abstract}
.
\end{abstract}

\begin{abstract}
RENE URBINA VERDUGO
Ex Director del D. Urbanismo - F.A.U. U. Chile, 1967-1973

[1959] "La planificación urbana y rural, incluyendo planificaciones especializadas como la habitacional, debe estructurarse descentralizadamente. Es recomendable, por ahora, atenerse a la división política administrativa oficial del país, en base de provincias y no de regiones, creando Oficinas de Planificación Urbana y Rural, semejantes a las Oficinas Provinciales de Arquitectura. Las Oficinas de provincias, debidamente financiadas con impuestos territoriales locales, constituirán la sede de las actividades urbanísticas en su jurisdicción y en ellas deben orientarse sistemáticamente los estudios previos y planes de las localidades menores, en colaboración con las municipalidades y con los Urbanistas particulares contratados como asesores de cada Plan Regulador local. Las ventajas de esta descentralización serán la posibilidad de realizar análisis y expedientes provinciales de planificación, válidos para varios centros poblados; la concentración técnica en un radio adecuado; mayor flexibilidad para la planificación, pasando por sobre los límites administrativos comunales inadecuados, etc. Además, la Oficina Provincial de Planificación coordinará el emplazamiento y oportunidad de las obras públicas fiscales".

[1957-58] "Resumiendo, exponemos [en el Seminario del Gran Santiago] como temas de discusión los siguientes interrogantes:

¿Qué bases económicas nacionales y regionales existen para aconsejar la detención del crecimiento demográfico de Santiago? ¿Existen otros medios prácticos para esta detención que no sea la paulatina descentralización industrial? ¿Cuáles son las posibilidades económicas y legales de descentralizar la industria? ¿Es indispensable descentralizarlas nacionalmente o bastaría regionalmente?

¿Si hay oposición de algunas municipalidades a un gobierno centralizador o coordinador de la metrópoli santiaguina, qué resortes deben tocarse para conseguirlo? ¿Debe la Dirección de Planeamiento integrarse con técnicos representantes de sus municipalidades. ¿Cómo puede obtenerse una mayor subdivisión administrativa de las comunas, manteniendo su unidad? ¿Existen condiciones propicias para la creación de un Ministerio de Vivienda y Urbanismo?

¿Es aconsejable la creación de unidades vecinales socialmente mixtas con participación de distintas instituciones en un mismo proyecto de conjunto? ¿Qué dificultades prácticas insalvables existirían para unificar, o por lo menos, coordinar efectivamente las obras de servicios públicos? ¿La remodelación de los sectores antiguos aumentará o disminuirá la capacidad demográfica de esas zonas?.

¿Cuáles son las vías que son de más urgente modificación para resolver los transportes masivos actualmente llevados a través del centro? ¿Cuál es el número de vehículos necesario para la movilización santiaguina y de qué tipo? ¿Cuáles son los organismos que deberían constituir un Comité para proponer medidas de fondo sobre el problema del tránsito? ¿Qué problemas o soluciones involucra la construcción del ferrocarril subterráneo?".

Fte.: Extracto 1, de: URBINA VERDUGO, René, "Estudio crítico general del plan Habitacional chileno desde el punto de vista urbanístico", en: UNIVERSIDAD DE CHILE, Facultad de Arquitectura, IVUPLAN, Departamento de Urbanismo, Algunos estudios de los aspectos urbanísticos del Plan Habitacional impulsado por el Supremo Gobierno en 1959, Ediciones del IVUPLAN, Santiago de Chile, 1959, 55 págs. (s / numeración).

Fte.: Extracto 2, de URBINA V., René, Relator en representación de la U. Chile, "Estudio comparativo de los problemas y soluciones urbanísticas de las grandes ciudades", en: UNIVERSIDAD DE CHILE - BOLETIN INFORMATIVO DEL DEPARTAMENTO DE EXTENSION CULTURAL, N³4, Añ́o III, octubre de 1958. Número especial dedicado a la $2^{a}$ y $3^{a}$ etapa del Seminario del Gran Santiago, 326 págs.; extracto en pp.34-38.
\end{abstract}




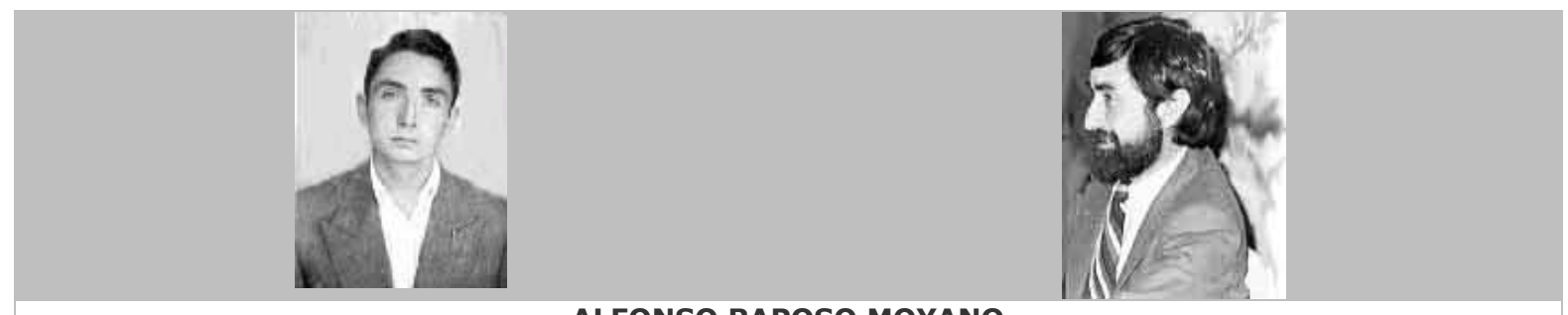

ALFONSO RAPOSO MOYANO

[1976] "Entendiendo el concepto de 'interés social' tanto en su significado teórico - político más idealmente virtuoso, como en su significado práctico - político más espurio, la perspectiva del interés social constituye la base originaria de las decisiones de la esfera política. En su referencia a la 'penuria de vivienda', la racionalización de la perspectiva del interés social ha sido, sin embargo, muy escasa. Las bases 'científicas' de la 'participación' permanecen en la ambigüedad y confusión tanto en las estrategias de dominación como en las estrategias contestatarias. La racionalización del interés social dentro del marco ideológico del universalismo democrático no parece contar con nada más que las 'técnicas de desarrollo de la comunidad' y el esfuerzo de los 'trabajadores sociales'. Las formas de implementación técnica del 'interés social' han seguido el camino de la racionalización formal en torno a nociones tales como: 'diálogo', 'asociación', 'mutualismo', 'corporativismo', 'cooperativismo', 'autogestión', etc. Sin alcanzar una ubicación clara en el ordenamiento institucional de la estructura de poder y la estructura de consumo. De aquí se concluye que, no obstante, ser el concepto de 'interés social' el más auténtico para servir de base a la articulación de información, es también el más impráctico tanto por la debilidad de la racionalización técnica de las formas de participación como por la naturaleza del momento socio-político predominante en América Latina".

Fte.: RAPOSO M., Alfonso, "Perspectivas analíticas en el diagnóstico y evaluación de la situación habitacional", en: GUROVICH, Alberto; RAPOSO, Alfonso, y VILLA, Miguel, Sistema Urbano y Vivienda, Documento de Trabajo $\mathrm{N}^{\circ} 16$, agosto de 1976, Departamento de Planificación Urbano-Regional, F.A.U. de la Universidad de Chile, p.75.

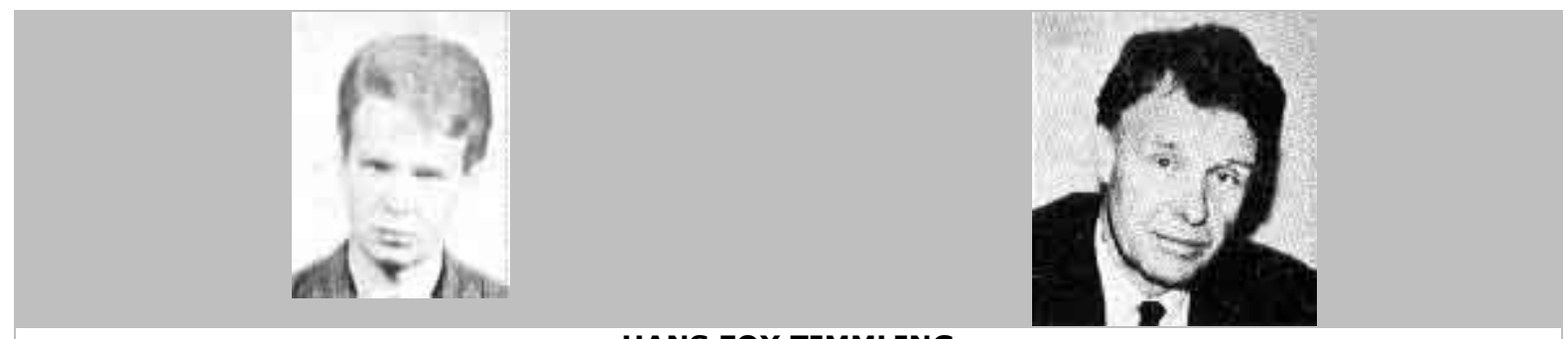

\section{HANS FOX TIMMLING}

[1970]"Podemos hablar de estética en el diseño urbano en la medida en que a través del diseño incorporemos en la ciudad comportamientos de realización. Al introducir comportamientos de realización en la ciudad, a través de las vivencias generamos espacios que significarán comunicación e intercambio humano. Al hacer cualquier modificación espacial en el medio urbano no hemos de contentarnos ya con esa modificación en sí misma, sino de preocuparnos de cuáles serán los comportamientos que estamos introduciendo o cuales perpetuaremos. Serán aquellos de realización, o aquellos de frustración, y son sólo los primeros los que nos transmutan hacia formas sociales de reunión humana. Hoy día el arquitecto no puede justificar o justificarse frente al medio con diseños que son logros estéticos en si mismos, desarticulados del medio ambiente. La tarea consiste en introducir en los espacios, a través de las formas, vivencias y comportamientos de realización. Así, el diseño urbano es fundamentalmente un asunto social. El fracaso de la arquitectura urbana actual es justamente su falta de dimensión social su incomprensión y aislamiento de la totalidad de la cual forma parte. Esta dimensión es la nueva escala contemporánea por conquistar, para poder concebir totalidades donde la ciudad ya no es una simple suma de partes. El diseño urbano tiene que trabajar con esta escala pues solo así logrará crear formas sociales de reunión, las cuales implicarán la obtención de vivencias de realización sensible. Teóricamente, el camino hacia el logro de vivencias de realización estaría despejado, pero prácticamente el problema subsiste, primero por una falta de conciencia social en los diseñadores, y segundo, por la ideología fundamentalmente antisocial de nuestras instituciones, ambos factores perpetúan un diseño antisocial."

Fte.: FOX, Hans, Las tensiones espaciales en el espacio urbano, tesis para optar al título de Arquitecto, Prof. Guía Arqto. Jorge Gómez R., 1970, págs. s/n. 


\section{VICENTE GAMEZ BASTEN}

[1973 (primer semestre)] "Si la planificación es un proceso social y la metropolización es un proceso social, el desarrollo y la transformación estructural de la sociedad habrá de provocar nuevas formas de organización social, nuevas leyes de relación social, de acumulación, de generación de excedente y sobreproducto; de regulación del intercambio, valoración y apropiación del producto social. Por esto, planificar no significa producir o prever situaciones futuras; por el contrario, planificar implica desarrollar la estructura social y puede significar, por lo tanto, "experimentar" nuevas formas organizacionales para asegurar el funcionamiento de las nuevas leyes de relación social. Estos experimentos sociales deben ser científicos, en el sentido de que deben estar orientados por una ideología científica, y sancionados históricamente por toda la sociedad. ¿Es factible un experimento social que reemplace la estructura urbana con leyes de mercado por una estructura social diferente? Hay precedentes de la "ruptura" de las leyes del mercado, por ejemplo, con las experiencias de estatización o de apropiación social del suelo urbano en forma total o parcial. Pero será sólo el desarrollo de una ciencia de la sociedad la que dará "respuestas" acerca de la realidad objetiva de una formación social concreta y de sus múltiples determinaciones estructurales. Ella permitirá también el desarrollo consecuente de una tecnología de la organización social que dé "soluciones", nuevas formas de relaciones técnicas y sociales para las asociaciones del futuro, permitiendo así desentrañar las múltiples interrogantes que se mantienen sobre el futuro de las grandes aglomeraciones metropolitanas y su rol en el proceso de desarrollo social".

Fte.: GAMEZ B., Vicente, "La metropolización de la sociedad y la planificación del fenómeno metropolitano", en revista de Planificación Vivienda Ciudad región, N8, Departamento de Estudios y Planificación UrbanoRegionales, F.A.U. de la Universidad de Chile, primer semestre de 1973, pp. 19-28.

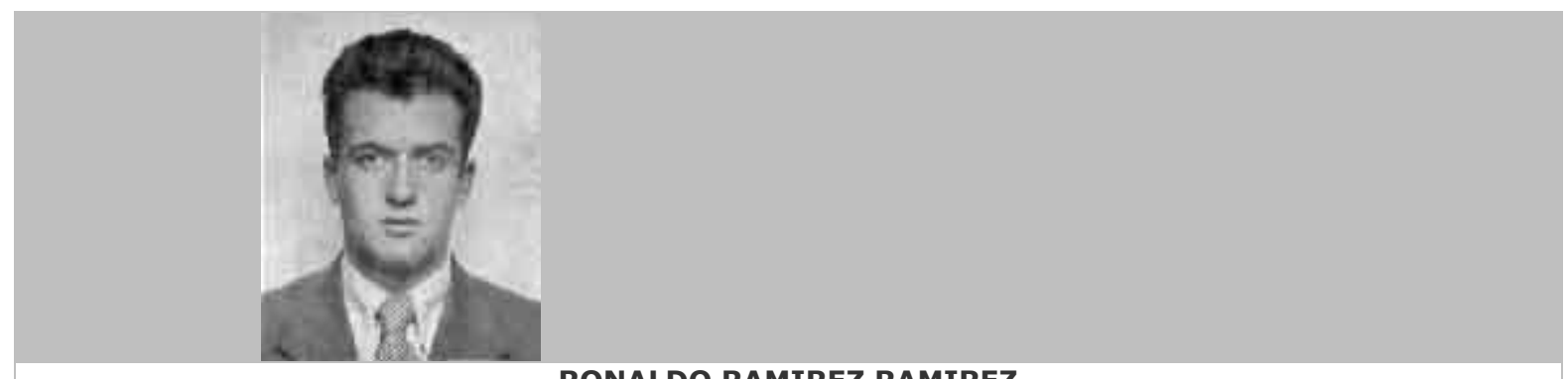

\section{RONALDO RAMIREZ RAMIREZ}

[1968] "En el caso de los países de América Latina, hay otra razón que atañe directamente a las universidades. En estos países las alternativas visibles para el desarrollo han sido encauzadas, mediante estrategias políticas, en dos vías principales que persiguen como objetivo el modelo de sociedades desarrolladas, sean capitalistas o socialistas. El agotamiento de los esquemas ideológicos que dan validez a estos modelos, y la cada vez más clara conciencia de sus debilidades y miserias, han empezado a descalificar estas alternativas. Además, la concepción misma del subdesarrollo ha llegado a evidenciar el carácter de dependencia que este estado implica en relación con las sociedades desarrolladas (Sunkel), lo que deja fuera de cuestión la superación de estas condiciones manteniendo las leyes del sistema. De esta manera, se plantea a los sectores intelectuales de estos países emprender la tarea de elaborar los modelos y las alternativas propias que permitan superar la actual situación. Modelos que para ser válidos no sólo tendrán que construirse con la experiencia universal, sino tendrán que fundarse en la realidad esencial de estos pueblos y su historia. Suponer que esta tarea se puede emprender al margen del conocimiento científico significaría reconocer la imposibilidad de su realización. Aparte de la formulación política que esto pueda traer, será la investigación en la realidad Latinoamericana, en su historia, en sus hombres, sus recursos, su potencialidad, etc., la mejor herramienta para la formulación de los modelos. Las instituciones mejor preparadas para realizar esta labor son las universidades de la región".

Fte.: RAMIREZ R., Ronaldo, "La crisis universitaria: contenido y estructura para una universidad comprometida", en: REVISTA DE PLANIFICACION, Vivienda Ciudad Región, N6, 1968, IVUPLAN, F.A.U. Universidad de Chile, pp. 35-72. 


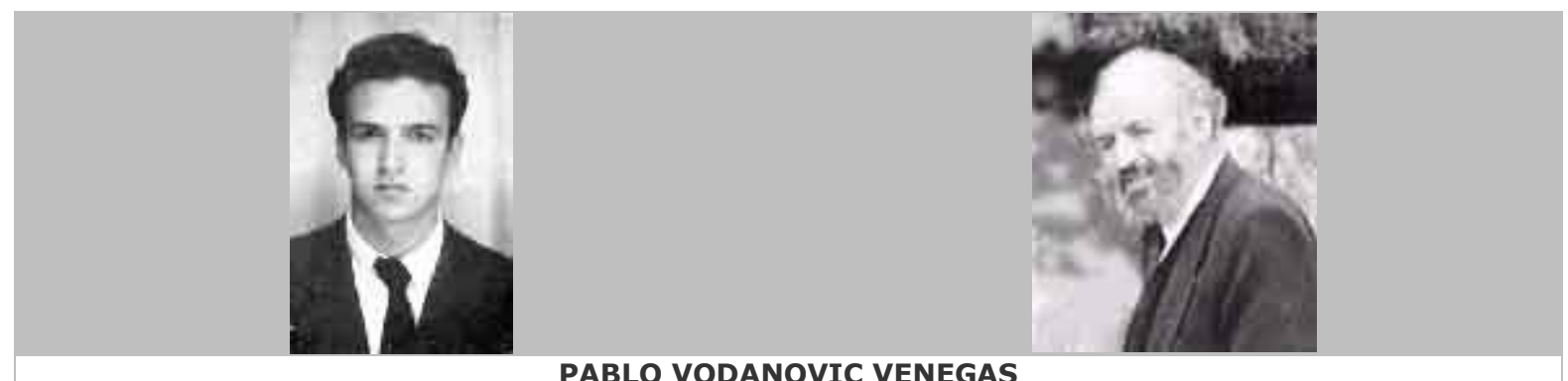

[1974-75] Nos permitimos insinuar que para los actuales intentos por crear un mercado de relativa libre competencia para la vivienda -dentro de lo que se ha denominado "una economía social de mercado", en el cual se estimula la libre demanda efectiva, resulta altamente necesario para el potencial comprador individual y colectivamente considerado -, el contar con elementos que le permitan evaluar los productos que en materia de vivienda brinda la oferta del sector productivo. Tanto las compras de viviendas terminadas efectuadas directamente por las personas naturales, como aquellas que se realizan a través de organismos o instituciones públicos y privados, deben apoyarse en criterios y evaluaciones objetivas y ajustadas de las viviendas y de los beneficios efectivos que el habitante obtendrá en términos de calidad, algunos de cuyos aspectos, como por ejemplo la durabilidad, determinan o comprometen a futuro la inversión pública y privada. Otro aspecto que sugerimos revisar desde el punto de vista cualitativo se refiere al concepto de déficit habitacional y a las políticas de vivienda basadas en el uso de este instrumento, posición que ha sido objeto de diversas críticas. En efecto, una política basada en la noción de déficit habitacional busca encontrar y poner en acción las medidas para suplir dicho déficit mediante la producción de unidades de habitación (casas). Para estimar este déficit se parte en realidad de conceptos cualitativos, pero tosca y discutiblemente aplicados. Se establece, por ejemplo, un límite cualitativo -la vivienda "aceptable"- bajo el cual todas las demás viviendas se consideran deficitarias. Al ignorar los aspectos sociales y culturales y la amplia gama de cualidades que pueden distinguirse en la vivienda, se termina por incurrir en gruesos errores de apreciación e imponiendo tácitamente a toda la población un modelo de habitación en forma arbitraria. Como consecuencia de esto se compromete la inversión pública y se orienta a la privada sin vislumbrar siquiera los efectos y los reales beneficios que se obtendrán de los recursos movilizados. Este tipo de enfoque, por las mismas causas, se caracteriza además, por comunicar la impresión de que la falta real de viviendas es inmensa y crece continua e inconmensurablemente, por lo cual toda acción habitacional aparece rodeada desde la partida de un halo de ineficiencia radical. Un planteamiento más sólido sólo puede lograrse a través de un manejo más adecuado y solvente de los aspectos cualitativos de la vivienda, para lo cual es imprescindible acrecentar el conocimiento que de ellos se tiene, al menos en los niveles de decisión de los organismos públicos. La evaluación cualitativa, sus conceptos, métodos e instrumentos, no son algo que, por otra parte, pueda resolverse de una vez y para siempre. Como se ha mostrado, se trata más bien de un esfuerzo constante y en permanente evolución y superación que debe cultivarse continuamente para ir paulatinamente mejorarlo y haciendo más efectivas y ajustados dichos conceptos, métodos e instrumentos.

Fte.: VODANOVIC, Pablo, Un programa de investigación en vivienda. Documento de Trabajo s/n, Departamento de Planificación Urbano-Regional, F.A.U. de la Universidad de Chile, pp.12-14, s/f. (c. 197475). 


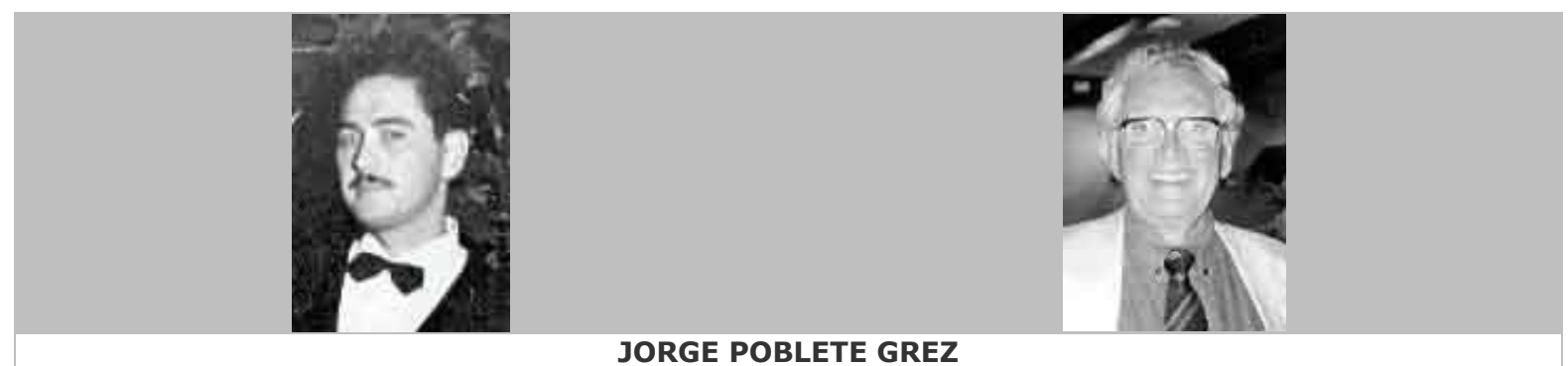

[1957-58] "Al término de su trabajo, la Comisión [Equipamiento Institucional, Seminario del Gran Santiago] cree haber podido constatar que:

-Existen muy escasos estudios sobre equipamiento el Gran Santiago, lo que se aprecia en el desorden de su organización y radicación.

-Los estudios conocidos por la Comisión son inconexos y en ciertos casos hay dualidad o paralelismo.

-Es indispensable para abordar integralmente el estudio y reducción de los problemas del Gran Santiago, que se elabore previamente un criterio de Planificación para el Gran Santiago, considerando, como un complejo urbano, en relación con el país y su región.

Es necesario crear un organismo de administración de la ciudad, que considere la comuna de Santiago y las demás que integran el Gran Santiago, como un solo conglomerado. Se debe dotar a dicho organismo de los recursos técnicos financieros y legales que le permitan encarar los complejos fenómenos de una urbe con absoluto realismo y amplia visión hacia el futuro. Se hace sentir con gran fuerza la necesidad de crear en el seno de la Universidad un organismo destinado a la formación de técnicos y a la investigación en materias de planeamiento".

[1966] "Iquique Región y Acción. Plan Regulador Comunal. Proyecto Específico: Plan Cordillera. Pretende reconstruir las estructuras sociales primitivas a fin de incorporarlas a la comunidad contemporánea, cuidando de no distorsionar sus valores históricos. Como planes específicos se propone: Agrícola. Elevar el nivel económico mediante la aplicación, de mejoras técnicas de explotación. Extensión de las áreas cultivadas a sus antiguos límites y en lo posible, superarlas. Artesanal. Recuperar las viejas artesanías para que sirvan de base a una industria de exportación extraregional. Cultural y Educacional. Elevar el nivel reforzando la herencia folklórica y cultural de estas antiguas comunidades. Para la realización de estos planes se organizarán las comunidades en sistemas cooperativos de producción y consumo".

Fte. de extracto 1.-: POBLETE G., Jorge, Presidente Comisión N9: Equipamiento institucional, "Informe de la Comisión No9 Equipamiento Institucional", en: UNIVERSIDAD DE CHILE - BOLETIN INFORMATIVO DEL DEPARTAMENTO DE EXTENSION CULTURAL, N³4, Año III, octubre de 1958. Número especial dedicado a la $2^{a}$ y $3^{a}$ etapa del Seminario del Gran Santiago, 326 págs.; extracto en pp.168-169.

Fte. De extracto 2.-: POBLETE G., Jorge, "Iquique Región y acción: Región de Iquique", en Revista $A U C A$ No5, 1966, pp. 57-59. 


\section{RUVSTADES URBANISMO

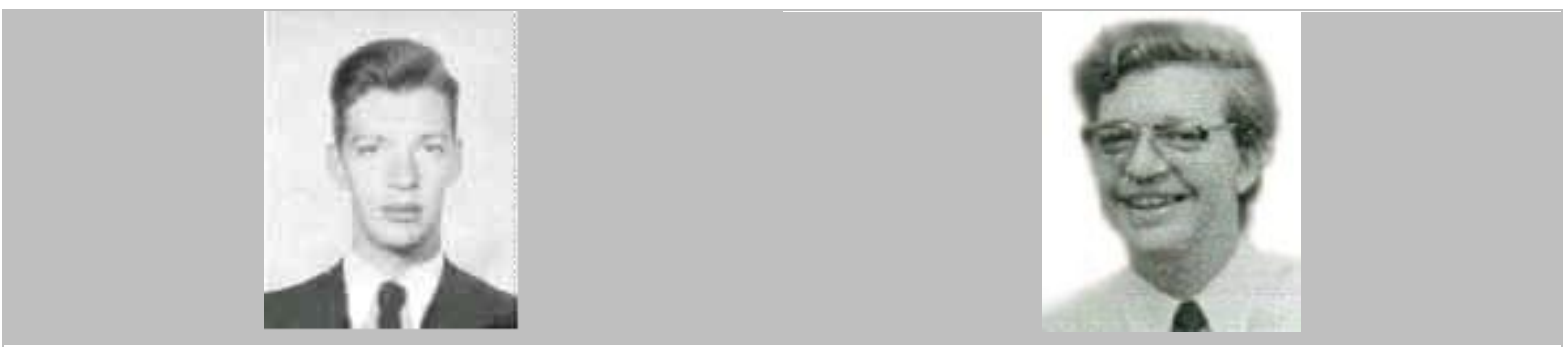

\section{PATRICIO ALZAMORA KURTH}

[1967] "Se precisa una transformación idónea del gobierno municipal, agregando eficiencia en su gestión sin vulnerar su valor de representativo directo de la comunidad; la planificación urbano-regional superará las condiciones locales, imprimiendo a su acción un carácter impulsor del desarrollo global. La mayoría de la población habita en áreas urbanas, proceso que tiende a intensificarse, por lo cual el progreso urbano será una condición indispensable del desarrollo social y económico nacional. La planificación coordinada de todos los niveles dará una visión integral de la problemática del desarrollo y combinará los esfuerzos urbanos con los de la región y el país. De este modo las políticas nacionales de vivienda y desarrollo urbano, entre otras, considerarán las particularidades locales en un amplio marco de referencia que contemple los posibles cambios de orientación económica y social del país, conjugando las decisiones de los diferentes niveles y sectores. No lesiona la autonomía municipal el compartir su acción frente a materias que, dada su magnitud técnica y financiera, requieren enfoques regionales o nacionales".

Fte.: ALZAMORA K., Patricio, Planificación del desarrollo Urbano-Regional, tesis para postular al título de Arquitecto, IVUPLAN, F.A.U. de la Universidad de Chile, Prof. Guía: Arqto. René Urbina Verdugo, 1967, p.TT-C3 PA/26-27.

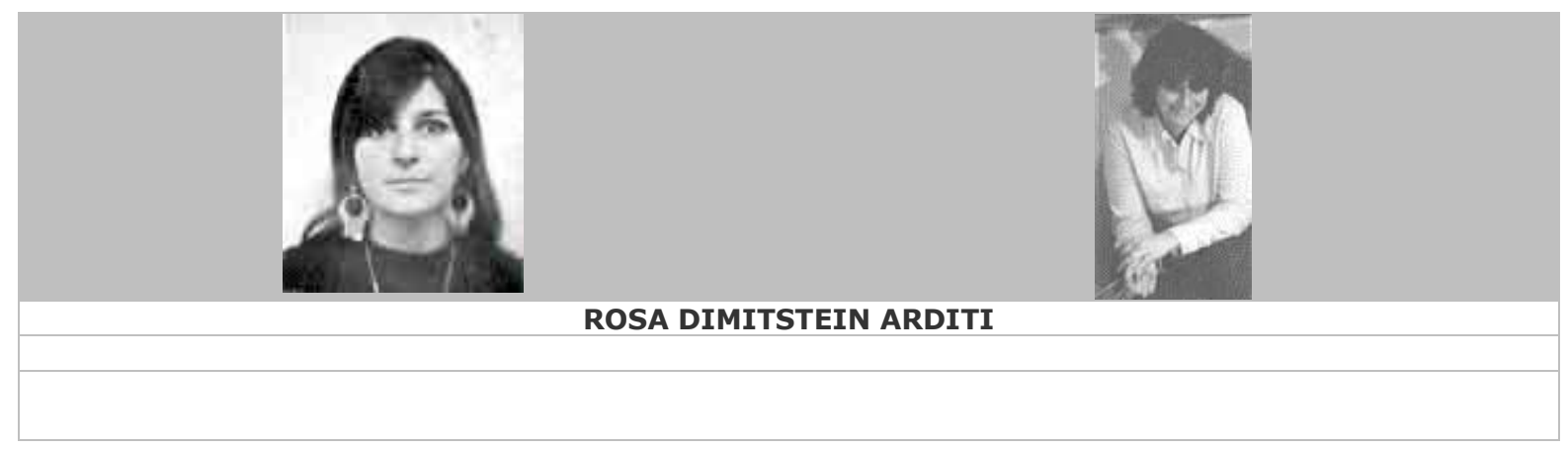




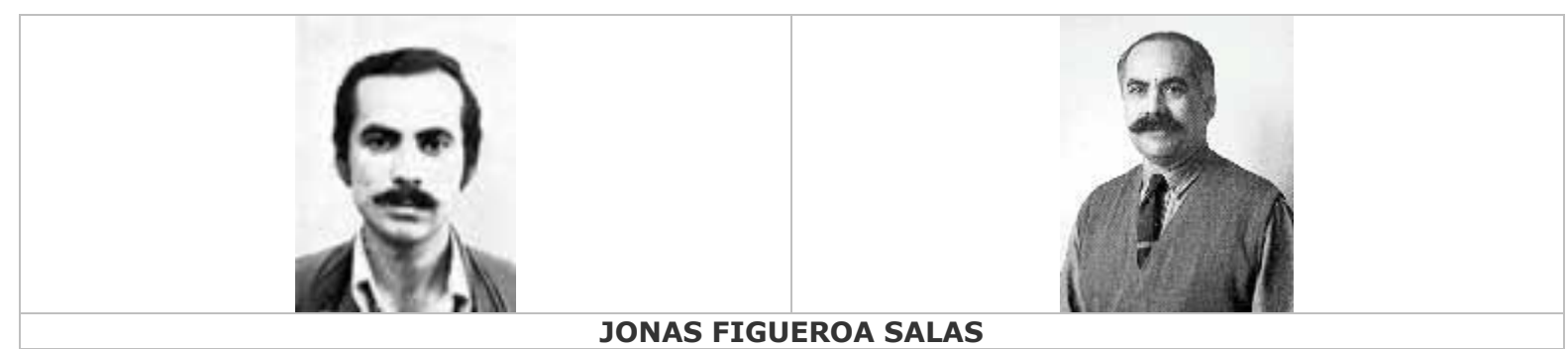

[1997] "Lo que hay: - el plan regulador es un mecanismo administrativo y predictivo, pero con una alta sujeción a las tendencias que exhibe el crecimiento real; - el contenido sólo promueve un trazado arterial que genera una ciudad al más puro estilo de obra pública, $y$, por último, - el plan regulador es incapaz de promover las necesarias transformaciones cualitativas que requiere la dimensión urbana, y, aún más, manifiesta un total divorcio con las grandes operaciones inmobiliarias. Lo que falta: el plan debe ser un mecanismo político y prospectivo que formule el proyecto urbano; - el plan también debe resolver la propuesta morfológica mediante el diseño de los elementos esenciales constituyentes de ciudad: centro, perímetro, piezas de articulación, corredores, etc., $y$, por último, - el planeamiento debe motivar la construcción de la ciudad, y definir por pura lógica existencial las grandes operaciones de transformación de ésta. Los nuevos suelos urbanos se incorporan a la ciudad a partir de una acción urbanística y se plasman bajo una expresión arquitectónica. Los efectos que tiene la ausencia de un dominio sobre la manufactura final (...), señalan la conveniencia de incorporar instrumentos que formulen la estructura morfológica del planeamiento. A partir de ello, el profundo abismo que separa la norma urbanística de la forma arquitectónica es salvado mediante una propuesta de arquitectura urbana".

Fte.: FIGUEROA S., Jonás, "Forma Urbis. La estructura Morfológica de la ciudad", en Revista CA N91, Ciudad/Arquitectura, ISSN 0716-3622, pp.78-83.

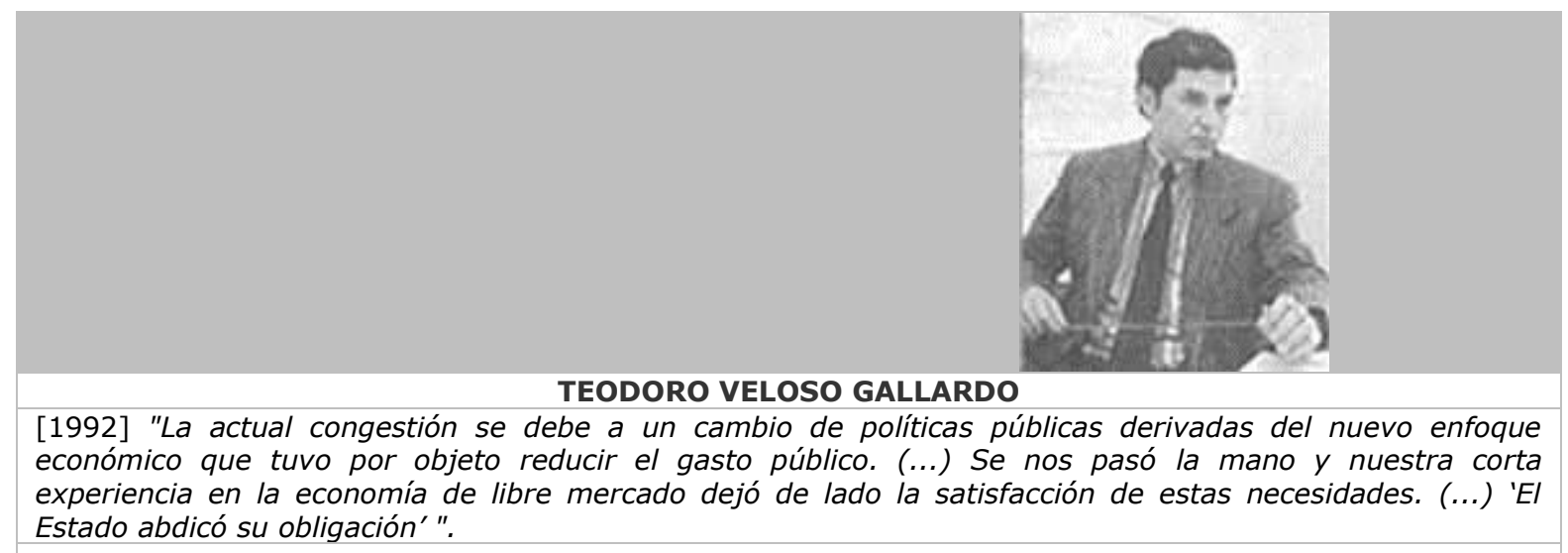

Fte.: Extracto de entrevista en diario ESTRUCTURA, Santiago, 15 de junio de 1992, p.8. 


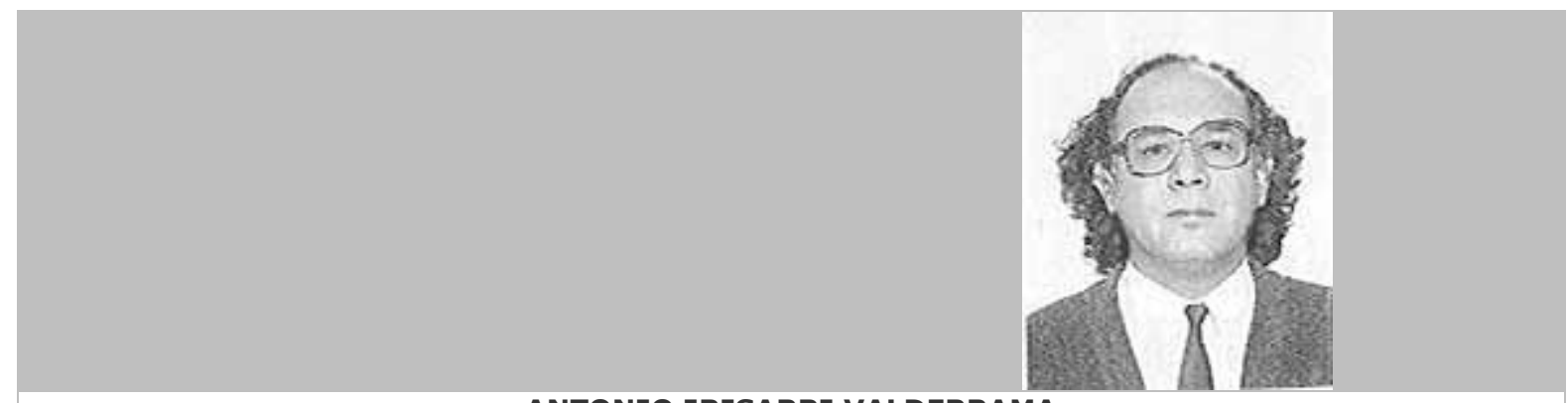
ANTONIO IRISARRI VALDERRAMA

[1971] "Haciendo una breve evaluación de lo que significó este trabajo [aplicación de información computarizada para desarrollo urbano], podemos concluir que la realidad estuvo muy lejos de las expectativas propuestas. El implementar un trabajo de creación de archivos de información y el procesamiento electrónico de datos, presupone un conocimiento profundo de la realidad de la información por capturar y un proceso acabado de adecuación de programas de computación a la calidad en que se encontrará esa información. No obstante ser esta una gran experiencia para el futuro, creemos que debió implementarse el uso de sistemas computacionales de proceso y captura de información en una ciudad con no más de 50.000 habitantes, para poder evaluar realmente los requerimientos técnicos que se necesitarán, las posibles formas en que se encontrará la información en terreno, etc. Esto habría aligerado los costos de una experiencia que, aunque relevante, creemos fue cara. Las evaluaciones sobre las posibilidades de obtener este archivo, hechas por la firma asesora del sistema, no previeron la verdadera realidad que se iba a encontrar. Esta firma extranjera de asesoría, no logró conocer a fondo el sistema administrativo y burocrático chileno, e hizo la evaluación bajo los mismos esquemas aplicados por ellos en otros países a los que han asesorado, como es el caso de Brasil, entre otros."

Fte.: IRISARRI V., Antonio; BURGOS, Libertad, Una aplicación de información computada para desarrollo urbano, Seminario de Investigación, Ciclo de titulación, IVUPLAN, F.A.U. Universidad de Chile, 1971, Prof. Guía Arqto. Pastor Correa P., pp.113.

\section{2.- Profesionales y Profesores del Urbanismo U. Chile S. XX}

Hemos querido también, hacer un recuerdo de los profesionales y profesores de muy diversas disciplinas, especialidades y escuelas, que han impartido docencia en diversos momentos históricos de nuestra Escuela y Facultad, por diversos períodos y tipos de actividad docente, tanto en el ciclo básico de la formación de Arquitecto, como en el de titulación, postítulo y posgrado, en Urbanismo.

Entre ellos:

Vicente ACUÑA, Lorenzo AGAR, Beatriz AGUIRRE, Jaime AHUMADA, Paulina AHUMADA, Horacio ALESSANDRINI, Lily ALTIMIRAS, Patricio ALZAMORA, José ARAYA, Mario ARAYA, Juan ARAYA, S. ARENSBURG, Gladis ARMIJO, Gabriela ARMIJO, Manuel ATAL, Manuel ATRIA.

Patricio BALBONTIN, Rodrigo BAÑo, Tomás de la BARRA, Ana. M. BARRENECHEA, Ivo BAVAROVIC, Moisés BEDRACK, Rolf BENQUE, Ximena BORQUEZ, J. BERMEJO, María 
BERTRAND, Irene BOISIER, Sergio BOISSIER, Alberto BOTTESELLE, Amador BRIEVA, Enrique BROWNE, Karl BRUNNER, Reginal BUDD, Libertad BURGOS.

Osvaldo CACERES, Ernesto CALDERÓN, Marcela CAMPOS, Luz A. CARDENAS, Luis CAPURRO, Gustavo CARRASCO, Sonia CASTRO, Hugo CONTRERAS, Raúl CORNEJO, Pastor CORREA, René COVARRUBIAS, Francisco CUMSILLE, Pedro CUNILL, Armando CHOMSKI.

Antonio DAHER, Jaime DAROCH, Rosa DIMITSTEIN, Martín DOMINGUEZ, Juan DOMICELY, Pedro DONOSO, Martín DURAN, M. Eugenia DUCCI, Marcial ECHEÑIQUE, Holger EGUMFELDT-JORGENSEN, F. EHIJO, Jaime ESCOBAR, Alejandro ESCOBAR, Irma ESPINOZA, Humberto ESPINOZA.

Giulietta FADDA, Manuel FERNANDEZ, Francisco FERRANDO, Rodrigo FERRARO, Jonás FIGUEROA, Eusebio FLORES, Raúl FLORES, Christian FONFACH, Walter FOCH, Hans FOX, N. FRANK, Rodolfo FRERES, César FUENZALIDA, Carlos FUENSALIDA.

Sergio GALILEA, Vicente GAMEZ, Francisco GARCIA-HUIDOBRO, Jaime GARRETON, Alfredo GASTAL, Enrique GEBHARD, Elvira GUERRERO, Rodolfo GIADACH, Jorge GOMEZ, Luis GOMEZ, Ricardo GONZALEZ, Patricia GONZALEZ, Ventura GONZALEZ, Raúl GONZALEZ, Alberto GUROVICH, Jorge GUZMAN, Carlos GUTIERREZ, Javier GUTIERREZ.

Jaime HALES, Jill HAMBERG, Fernando HARTWIG, Raimundo HEDERRA, Patricia HENRIQUEZ, Helia HENRIQUEZ, José HENRIQUEZ, Carmen HEPP, Vladimir HERMOSILLA, Enrique HERNANDEZ, Amílcar HERRERA, Juan HONOLD, Julio HURTADO, Antonio INFANTE, Eliana ISRAEL, Kurt HERDAN, Antonio IRISARRI, Roberto IZAURIETA.

Alfredo JOHNSON, Marcos KAPLAN, Miryam KATTAN, Susana KORINFELD, Igor KRONEBERG, Fernando KUSNETZOFF, Fernando LARRAGUIBEL, Guillermo LEFORT, Alejandro LEON, George LEVER, Luis LIRA, Julio LOPEZ, Waldo LOPEZ, Antolín LOPEZ, Oscar MAC-CLURE, Hugo MADARIAGA, Florencio MAGALLON, Héctor MARDONES, Pablo MARDONES, M. Eliana MARTINEZ, Jorge MARTINEZ, René MARTINEZ, Francisco MARTINEZ, Rodrigo MAYO, Ramón MENDEZ, J.A. MENDIZABAL, Julio MONREAL, Alejandro MONTERO, Miguel MORALES, Sergio MORALES, Emilio MOYANO, Gustavo MUNIZAGA, Julio MUNIZAGA, Carlos MUNIZAGA, Carlos MUÑOZ, Luis MUÑOZ, Mauricio MUÑOZ, Sergio MOREIRA, Aquiles MORENO.

Héctor NAVARRETE, Eduardo NECOCHEA, Max NUÑEZ, Federico OEHRENS, Enrique OVIEDO, Jacqueline OXMAN, Rodulfo OYARZUN, Juan OYOLA, Martha PALMA, Licio PARISI, Juan PARROCHIA, M. Isabel PAVEZ, Mario PERALTA, Rafael PINO, Javier PINTO, Carlos PLAZA, Ricardo POBLETE, Jorge POBLETE, Marcia POUPIN, Patricio de la PUENTE, Gabriel PUMARINO, Aníbal QUIJANO.

Ronaldo RAMIREZ, Oscar RAMOS, Alfonso RAPOSO, Sebastián RIEDE, Margarita RIFFO, Mario RIQUELME, Carlos RIQUELME, Hernán RIVERA, Eduardo ROJAS, Antonio 
RODRIGUEZ-CANO, Enrique ROMAN, Hugo ROMERO, Mario ROSALES, Jurgen ROTTMANN, Adriano ROVIRA.

Hugo SAA, René SAA, Hernán SAN MARTIN, Alberto SCHADE, Antonio SAHADY, Mariana SALCEDO, Fernando SALAMANCA, Carlos SANHUEZA, Ignacio SANTA MARIA, Henrich SASENFELD, Martha SCHTEINGART, Sergio SEELEMBERG, Fernando SILVA, Julio SILVA, Jaime SILVA, Mark SMITH, Aldo SOLARI, Antonieta SURAWSKI.

Astolfo TAPIA, Mario TORRES, Pablo TRIVELLI, Francisco UNDA, Guillermo ULRIKSEN, Pablo ULRIKSEN, Eliana URANGA, René URBINA, Silvia URRUTIA, Rubén UTRIA.

Carlos VALDEBENITO, Juan VALENZUELA, Fernando VALDERRAMA, Alfredo VALLADARES, Carlos VARAS, Alejandro VARGAS, Álvaro VASQUEZ, Gabriel VEGA, Teodoro VELOSO, José VERA, Oscar VERA, Miguel VILLA, Francisco VILLASEÑOR, Marta VIVEROS, Pablo VODANOVIC, Carlos WEBER, Pedro YAGNAM, Eduardo ZAPATER, Enrique ZÁRATE, Herman ZEPEDA.

Hacemos extensivo este homenaje a los equipos de ayudantes de quienes tuvieron la responsabilidad directa de la tarea docente, y que, con igual generosidad, proporcionaron su tiempo, paciencia y conocimientos no sólo en la labor docente, sino también en el desarrollo de las labores creativas de la Universidad de Chile. Por razones de tiempo y restricciones en las fuentes, no es posible reconstituir este listado de profesionales.

\section{Profesionales del Urbanismo visitantes}

De igual forma, al concluir el siglo XX, hacemos un recuerdo de los profesionales y profesores del Urbanismo visitantes, venidos desde diversos continentes, y cuya presencia ha significado un estímulo trascendente en nuestra casa de estudios superiores ${ }^{2}$.

Entre ellos:

\footnotetext{
${ }^{2}$ Las fotografías que acompañan este listado se han encontrado en los archivos del Departamento de Historia y Teoría de la Arquitectura, y de la Unidad de Extensión, de la F.A.U. de la Universidad de Chile; en las revistas francesas METROPOLIS, Y URBANISME, por gentileza del Director de esta última, el Sr, Bernard Ëcrement; en REVISTA CA, órgano Oficial del Colegio de Arquitectos de Chile; en los archivos de la Akademie für Raumsforchung und Landes Planung, de Hannover, por gentileza de su Director el Dr. K. Becker; en el diario "EL Mercurio" de Santiago, y en archivos privados.
} 
Entre 1900 y 1939: Jacques LAMBERT, Karl BRUNNER. En los años 1940s, Gaston BARDET.

En los años 1950s: Luis ORTIZ DE ZEBALLOS, William REX CRAWFORD, Kurt BRÜNING; Eugene BEAUDOUIN, Aarón HORWITZ, Cornelis Van EESTEREN.

En los años 1960s: Rudyard JONES; Paul BRENIKOV; Julius GORINSKY; Lawrence MANN; Kurt SIEGEL; Félix CANDELA; Charles FRANKENHOFF; Francis VIOLICH; John A. PARKER.

En los años 1970s: Heinrich K. SASENFELD, Armando CHOMSKI, Samuel SCKAKED, Bernard KAISER, Henri LEFEBVRE, Roberto SEGRE, Fernando SALINAS, John LEONARD, Regina de TOLEDO, Richard MORSE, John DYCKMAN, Richard LE GATES; Stephen COHEN, Colin BALMER, Francis VIOLICH, Douglas B. LEE.

En los años 1980s: Irene de AVELLANEDA, Francisco FERNANDEZ LONGORIA, Hans P. BAHRDT, Bruno VAYSSIERE, Klaus BORCHARD, Michael TRIEB, Phillipe PANERAI, Alexander SCHMIDT; Ivor SAMUELS; Lucía BRIONES; Karolus HEIL; Theo KÖTHER; Bernd STREICH, John MARTIN LINDSAY, Hervé BLUMENFELD, Peter TREUNER, Robert WISCHER, Michel MAFFESOLI, Horacio TORRES, Gabriel DUPUY.

En los años 1990s: Max PEDEMONTE, Michael MATTINGLY, Jonathan BARTON, Michael TRIEB, Klaus BORCHARD, David GOODE, Urbano RIFATERRA, Antoni PUJOL, Jonathan BARTON, Mike BRADSHAW, Nigel HARRIS, Claude BATAILLON, Bill HILLIER, Anders MALM, Silvio CACCIA, Andreas HOFER, Fritz ÖTTL, Phillipe PANERAI, M. Josefina FLOREZ, Gary D. MC CALEB, Pedro ORTIZ, Javier de MESONES, Marcial ECHEÑIQUE, Francesc MAGRINYA.

\section{Galería de retratos}

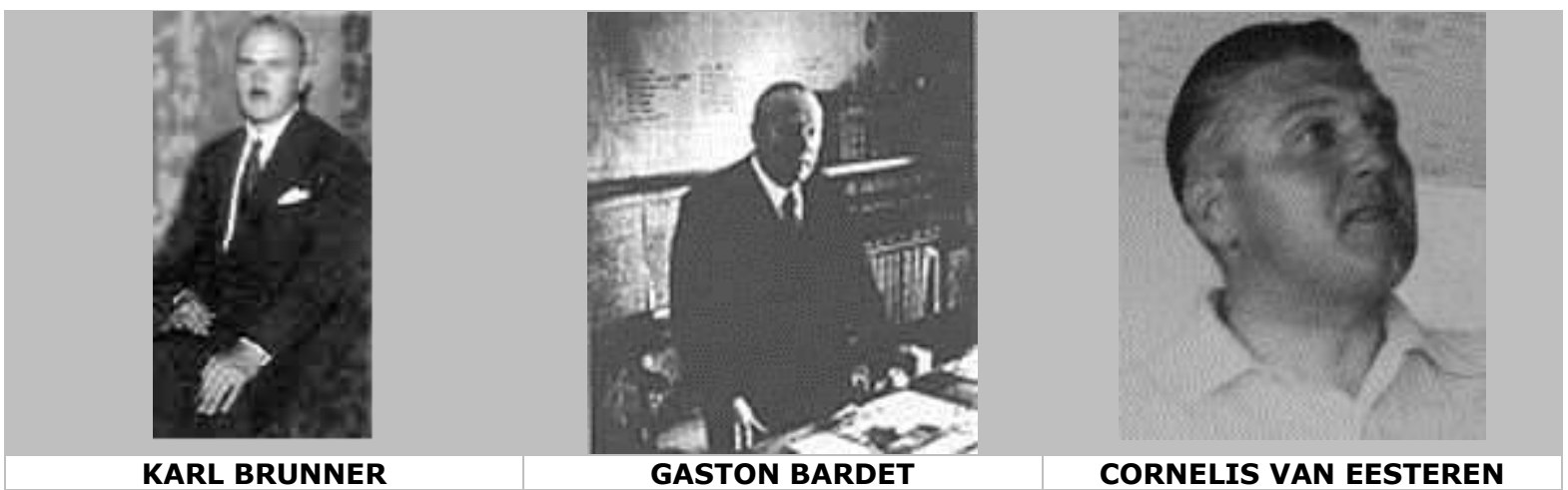




\section{REVISTA DE URBANISMO

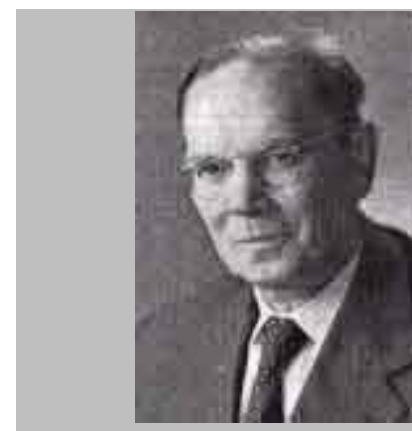

KURT BRÜNNING

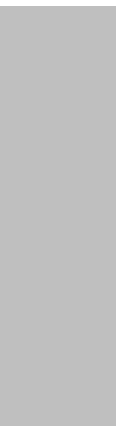

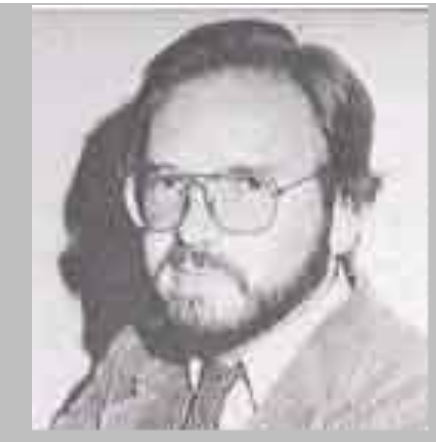

F. FERNÁNDEZ LONGORIA

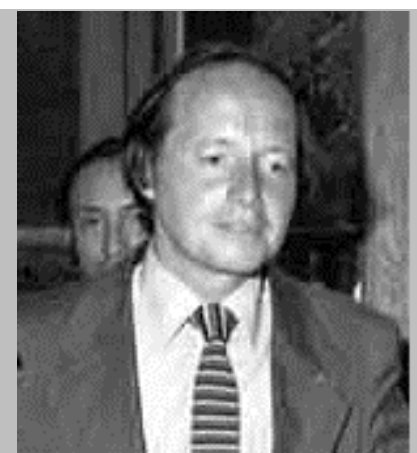

KLAUS BORCHARD
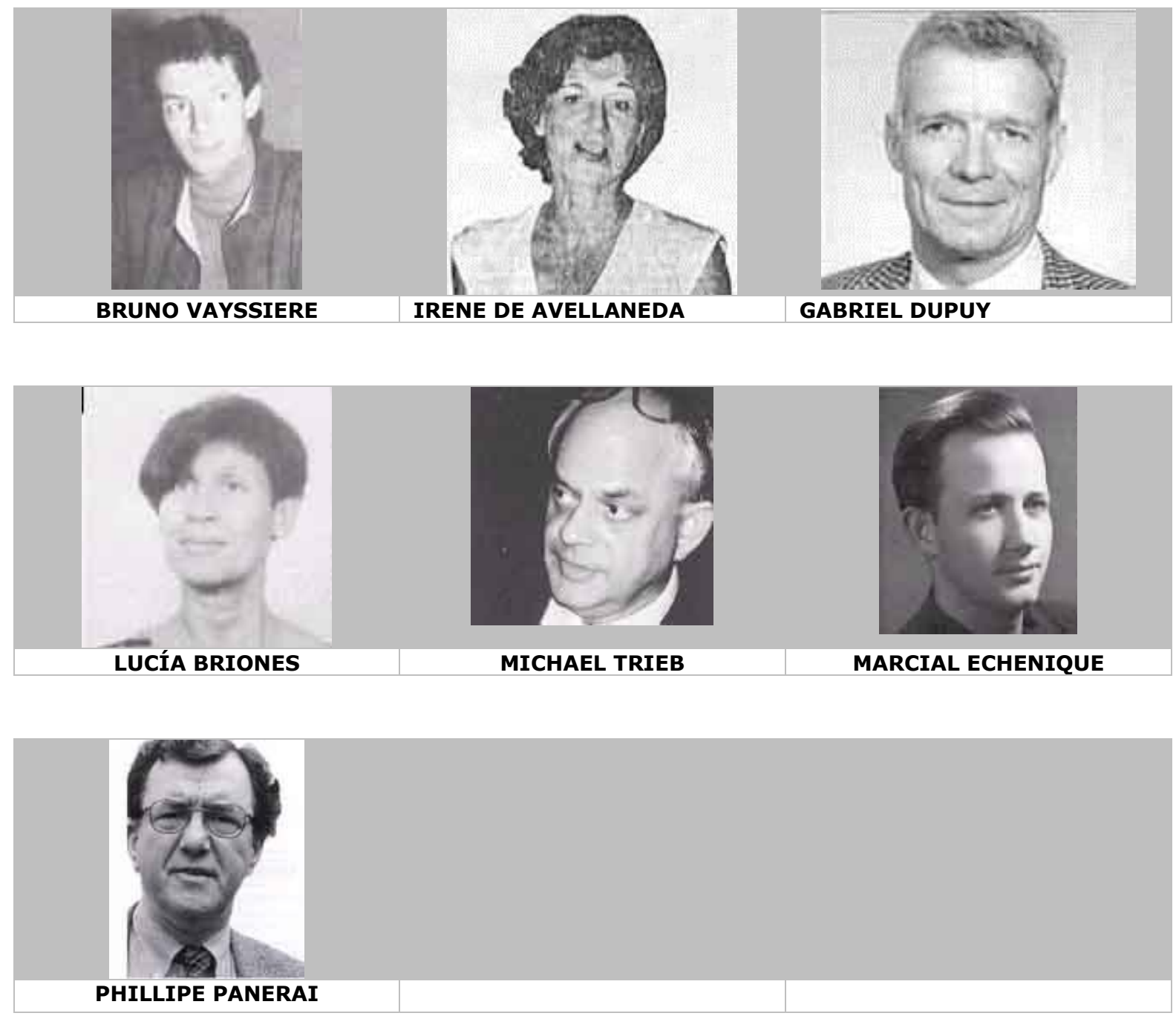